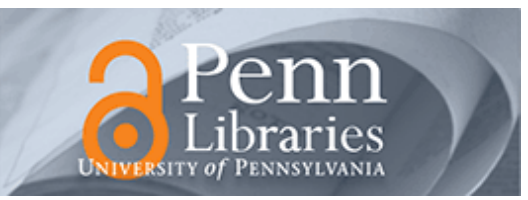

University of Pennsylvania

ScholarlyCommons

\title{
Guided Modes in a Waveguide Filled With a Pair of Single- Negative (SNG), Double-Negative (DNG), and/or Double-Positive (DPS) Layers
}

\author{
Andrea Alù \\ University of Pennsylvania, andreaal@seas.upenn.edu \\ Nader Engheta \\ University of Pennsylvania, engheta@seas.upenn.edu
}

Follow this and additional works at: https://repository.upenn.edu/ese_papers

Part of the Electrical and Computer Engineering Commons

\section{Recommended Citation}

Andrea Alù and Nader Engheta, "Guided Modes in a Waveguide Filled With a Pair of Single-Negative (SNG), Double-Negative (DNG), and/or Double-Positive (DPS) Layers", . January 2004.

NOTE: At the time of publication, author Andrea Alù was affiliated with the University Roma Tre. As of September 2006, she is a staff member in the Electrical and Systems Engineering Department at the University of Pennsylvania. Copyright (C 2004 IEEE. Reprinted from IEEE Transactions on Microwave Theory and Technniques, Vol. 52, No. 1, January 2004 Publisher URL: http://dx.doi.org/10.1109/TMTT.2003.821274

This material is posted here with permission of the IEEE. Such permission of the IEEE does not in any way imply IEEE endorsement of any of the University of Pennsylvania's products or services. Internal or personal use of this material is permitted. However, permission to reprint/republish this material for advertising or promotional purposes or for creating new collective works for resale or redistribution must be obtained from the IEEE by writing to pubs-permissions@ieee.org. By choosing to view this document, you agree to all provisions of the copyright laws protecting it.

This paper is posted at ScholarlyCommons. https://repository.upenn.edu/ese_papers/2

For more information, please contact repository@pobox.upenn.edu. 


\title{
Guided Modes in a Waveguide Filled With a Pair of Single-Negative (SNG), Double-Negative (DNG), and/or Double-Positive (DPS) Layers
}

\begin{abstract}
Here we present the results of our theoretical analysis for guided modes in parallel-plate waveguides filled with pairs of parallel layers made of any two of the following materials: 1) a material with negative real permittivity, but positive real permeability (epsilon-negative); 2) a material with negative real permeability, but positive real permittivity (mu-negative); 3 ) a material with both negative real permittivity and permeability (double-negative); and 4) a conventional material with both positive real permittivity and permeability (double-positive) in a given range of frequency. Salient properties of these guided modes are studied in terms of how these materials and their parameters are chosen to be paired, and are then compared and contrasted with those of the guided modes in conventional waveguides. Special features such as monomodality in thick waveguides and presence of TE modes with no-cutoff thickness in thin parallel-plate waveguides are highlighted and discussed. Physical insights and intuitive justifications for the mathematical findings are also presented.
\end{abstract}

\section{Keywords}

Double-negative (DNG) material, left-handed (LH) material, metamaterial, negative index material, negative index of refraction, negative permeability, negative permittivity, waveguide

\section{Disciplines}

Electrical and Computer Engineering

\section{Comments}

NOTE: At the time of publication, author Andrea Alù was affiliated with the University Roma Tre. As of September 2006, she is a staff member in the Electrical and Systems Engineering Department at the University of Pennsylvania. Copyright @ 2004 IEEE. Reprinted from IEEE Transactions on Microwave Theory and Technniques, Vol. 52, No. 1, January 2004 Publisher URL: http://dx.doi.org/10.1109/ TMTT.2003.821274

This material is posted here with permission of the IEEE. Such permission of the IEEE does not in any way imply IEEE endorsement of any of the University of Pennsylvania's products or services. Internal or personal use of this material is permitted. However, permission to reprint/republish this material for advertising or promotional purposes or for creating new collective works for resale or redistribution must be obtained from the IEEE by writing to pubs-permissions@ieee.org. By choosing to view this document, you agree to all provisions of the copyright laws protecting it. 


\title{
Guided Modes in a Waveguide Filled With a Pair of Single-Negative (SNG), Double-Negative (DNG), and/or Double-Positive (DPS) Layers
}

\author{
Andrea Alù and Nader Engheta, Fellow, IEEE
}

\begin{abstract}
Here we present the results of our theoretical analysis for guided modes in parallel-plate waveguides filled with pairs of parallel layers made of any two of the following materials: 1) a material with negative real permittivity, but positive real permeability (epsilon-negative); 2) a material with negative real permeability, but positive real permittivity (mu-negative); 3) a material with both negative real permittivity and permeability (double-negative); and 4) a conventional material with both positive real permittivity and permeability (double-positive) in a given range of frequency. Salient properties of these guided modes are studied in terms of how these materials and their parameters are chosen to be paired, and are then compared and contrasted with those of the guided modes in conventional waveguides. Special features such as monomodality in thick waveguides and presence of TE modes with no-cutoff thickness in thin parallel-plate waveguides are highlighted and discussed. Physical insights and intuitive justifications for the mathematical findings are also presented.
\end{abstract}

Index Terms-Double-negative (DNG) material, left-handed (LH) material, metamaterial, negative index material, negative index of refraction, negative permeability, negative permittivity, waveguide.

\section{INTRODUCTION}

I N 1967, Veselago postulated theoretically a material in which both permittivity and permeability were assumed to have negative real values, and he analyzed plane-wave propagation in such a medium, which he called "left-handed" (LH) medium [1]. According to his analysis, in such a "double-negative" (DNG) [2] material, the Poynting vector of a plane wave is antiparallel with its phase velocity. In recent years, Shelby et al. [3], inspired by the work of Pendry et al. [4], [5], constructed a composite medium in the microwave regime, by arranging arrays of small metallic wires and split ring resonators [3], [6], [7], and they demonstrated the anomalous refraction for

Manuscript received April 17, 2003. This work was supported in part by the Fields and Waves Laboratory, Department of Electrical and Systems Engineering, University of Pennsylvania. The work of A. Alù was supported by the Associazione Elettrotecnica ed Elettronica Italiana (AEI) under the Isabella Sassi Bonadonna Scholarship. This paper is an expansion of a paper presented at the 2003 IEEE Microwave Theory and Techniques Society (IEEE MTT-S) International Microwave Symposium.

A. Alù was with Department of Electrical and Systems Engineering, University of Pennsylvania, Philadelphia, PA 19104-6390, USA. He is now with the Department of Applied Electronics, University of "Roma Tre," 00146 Rome, Italy (e-mail: alu@uniroma3.it).

N. Engheta is with the Department of Electrical and Systems Engineering, University of Pennsylvania, Philadelphia, PA 19104-6390 USA (e-mail engheta@ee.upenn.edu).

Digital Object Identifier 10.1109/TMTT.2003.821274 this medium. Various aspects of this class of metamaterials are now being studied by several groups worldwide, and many ideas and suggestions for potential applications of these media have been mentioned (see, e.g., [1]-[52]). As one such idea, in some of our previous studies, we theoretically suggested the possibility of having thin subwavelength cavity resonators in which a layer of the DNG medium is paired with a layer of conventional material (i.e., a "double-positive" (DPS) medium) [36]-[38]. By exploiting the antiparallel nature of the phase velocity and Poynting vectors in a DNG slab, we theoretically found the possibility of resonant modes in electrically thin parallel-plate structures containing such DNG-DPS bilayer structures [36]-[38]. Following those studies, a first set of preliminary results and ideas for the guided modes in a parallel-plate waveguide containing a pair of DNG and DPS slabs was presented in [37] and [38]. Later in [40], we showed the effects of the anomalous mode coupling between DNG and DPS open waveguides located parallel to, and in proximity of, each other. Some other research groups have also explored certain aspects of waveguides involving DNG media. Among those, one may mention the work reported in [47]-[51].

Most of the work in the area of metamaterials reported in the recent literature has thus far been concerned with the wave interaction with DNG media. However, "single-negative" (SNG) materials in which only one of the material parameters, not both, has a negative real value may also possess interesting properties when they are paired in a conjugate manner. These media include the epsilon-negative (ENG) media, in which the real part of permittivity is negative, but the real permeability is positive, and the mu-negative (MNG) media, in which the real part of permeability is negative, but the real permittivity is positive. For instance, the idea of constructing an effective LH medium by having layers of SNG media has been explored by Fredkin and Ron in [46]. We have analyzed in detail the wave interaction with a pair of juxtaposed ENG and MNG slabs, showing interesting properties such as resonances, transparency, anomalous tunneling, and zero reflection [44]. Using appropriate distributed circuit elements in the transmission-line model for the pair of ENG-MNG layers, we have theoretically explained the unusual field behavior in these paired ENG-MNG structures and we have shown that such lossless pairs may exhibit "interface resonance" phenomena, even though each slab alone does not manifest such an effect [44]. Therefore, suitably coupled SNG media may offer exciting possibilities in the design of future devices and components, and since only one of their parameters needs to be negative in a given frequency range, it 
is conceivable that they may conceptually be constructed more easily than DNG media, for which both parameters should possess negative real parts in a given band of frequencies. For instance, a collisionless isotropic plasma, whose permittivity may be negative at frequencies below the plasma frequency [53] is, indeed, an ENG medium at those frequencies.

As a further contribution to the topic of wave interaction with SNG and DNG media, here we present the results of our theoretical analysis of parallel-plate waveguiding structures filled with pairs of layers made of any two of ENG, MNG, DPS, and DNG materials, exploring possible unconventional features that depend on the choice of the constitutive parameters. In our analysis, these materials are assumed to be lossless, homogeneous, and isotropic. In this paper, we show that by juxtaposing "conjugate" materials, i.e., materials with complementary electromagnetic properties, unusual features may be achieved in such guiding structures. For example, the possibility of monomodal propagation in arbitrarily thick parallel-plate waveguides filled with a pair of ENG-MNG layers and modes with no-cutoff thickness is highlighted. Although the structures considered here are the parallel-plate waveguides filled with SNG and DNG media, their features may indeed provide physical insights into exciting ideas and characteristics for other waveguide geometries filled with such metamaterials, with potential applications in the design of future devices and components.

A special note regarding the dispersive nature of these metamaterials is in order here. It is important to note that passive SNG and DNG metamaterials, in which permittivity and/or permeability may have negative real parts, are inherently dispersive [1], [42]. (The idea of active metamaterials in which negative parameters may conceptually occur over a relatively larger band of frequency is discussed by Tretyakov in [20].) Therefore, for passive metamaterials, the real parts of the material parameters may be negative only over a certain band of frequencies and, thus, their values may significantly vary with the frequency. As a result, one should, in general, take into account the frequency dependence of such material parameters. However, here, in order to emphasize the salient features of this type of waveguides without resorting to an unnecessary complexity, we fix the frequency of operation $\omega$ and we consider the values of permittivity and permeability of SNG, DNG, and DPS materials at this given frequency. All other parameters of the waveguide such as layer thicknesses and longitudinal wavenumbers may arbitrarily vary.

\section{GeOMETRY AND Formulation OF THE PROBLEM}

We consider a parallel-plate waveguide, made of two infinitely extent perfectly electric conducting plates separated by the distance $d=d_{1}+d_{2}$, as shown in Fig. 1 . This waveguide is filled with a pair of parallel layers made of any two of ENG, MNG, DNG, and DPS materials. A monochromatic time-harmonic variation $e^{j \omega t}$ is assumed. The two slabs are characterized by their thicknesses $d_{1}$ and $d_{2}$, and constitutive parameters $\varepsilon_{1}, \mu_{1}$ and $\varepsilon_{2}, \mu_{2}$, which are assumed real, but no assumption on their signs has yet been made. The Cartesian coordinate system $(x, y, z)$ is shown in Fig. 1 and $x$ is chosen as the direction of propagation of guided modes.

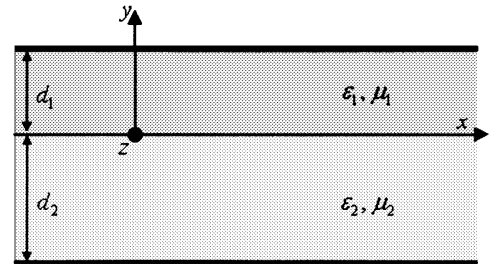

Fig. 1. Geometry of the parallel-plate waveguide filled with a pair of layers made of any two of ENG, MNG, DNG, and DPS materials.

Imposing appropriate boundary conditions at $y=d_{1}$ and $y=-d_{2}$, the electric- and magnetic-field expressions for the $\mathrm{TE}^{x}$ mode may be written as

$$
\begin{aligned}
\boldsymbol{E}^{\mathrm{TE}}= & \hat{z} E_{0}^{\mathrm{TE}} e^{-j \beta_{\mathrm{TE}} x} \\
& \cdot\left\{\begin{array}{ll}
\sin \left(k_{t 2}^{\mathrm{TE}} d_{2}\right) \sin \left[k_{t 1}^{\mathrm{TE}}\left(d_{1}-y\right)\right], \\
\sin \left(k_{t 1}^{\mathrm{TE}} d_{1}\right) \sin \left[k_{t 2}^{\mathrm{TE}}\left(y+d_{2}\right)\right], & y>0
\end{array} \quad\right. \\
\boldsymbol{H}^{\mathrm{TE}}= & j \hat{\boldsymbol{x}} \omega^{-1} E_{0}^{\mathrm{TE}} e^{-j \beta_{\mathrm{TE}} x} \\
& \cdot\left\{\begin{array}{l}
-\mu_{1}^{-1} k_{t 1}^{\mathrm{TE}} \sin \left(k_{t 2}^{\mathrm{TE}} d_{2}\right) \cos \left[k_{t 1}^{\mathrm{TE}}\left(d_{1}-y\right)\right] \\
+\mu_{2}^{-1} k_{t 2}^{\mathrm{TE}} \sin \left(k_{t 1}^{\mathrm{TE}} d_{1}\right) \cos \left[k_{t 2}^{\mathrm{TE}}\left(y+d_{2}\right)\right]
\end{array}\right. \\
& -\hat{\boldsymbol{y}} \omega^{-1} \beta_{\mathrm{TE}} E_{0}^{\mathrm{TE}} e^{-j \beta_{\mathrm{TE}} x} \\
& \cdot \begin{cases}\mu_{1}^{-1} \sin \left(k_{t 2}^{\mathrm{TE}} d_{2}\right) \sin \left[k_{t 1}^{\mathrm{TE}}\left(d_{1}-y\right)\right], & y>0 \\
\mu_{2}^{-1} \sin \left(k_{t 1}^{\mathrm{TE}} d_{1}\right) \sin \left[k_{t 2}^{\mathrm{TE}}\left(y+d_{2}\right)\right], & y<0\end{cases}
\end{aligned}
$$

where $E_{0}^{\mathrm{TE}}$ is the mode amplitude, determined by the excitation, and $k_{\mathrm{ti}}^{\mathrm{TE}}=\sqrt{k_{i}^{2}-\beta_{\mathrm{TE}}^{2}}$ with $k_{i}^{2}=\omega^{2} \mu_{i} \varepsilon_{i}$ for $i=1,2$. The corresponding expressions for the $\mathrm{TM}^{x}$ modes may be easily obtained (not shown here). For ENG and MNG slabs, where one of the material parameters is negative, we have $k_{i}^{2}<0$, and for propagating modes with real $\beta$, the transverse wavenumber $k_{\mathrm{ti}}$ is always imaginary. However, for DPS and DNG slabs, $k_{i}^{2}>0$ and the transverse wavenumber $k_{\mathrm{ti}}$ may be real or imaginary, depending on the value of $\beta$. The field expressions in (1) and (2) and the corresponding expressions for the TM case, however, remain valid for any of these cases. (For simplicity, heretoafter we drop the superscript " $x$ " in $\mathrm{TE}^{x}$ and $\mathrm{TM}^{x}$ ). By applying the boundary conditions for the tangential components of the electric and magnetic fields at the interface $y=0$, one finds the following two dispersion relations for the TE and TM modes, respectively:

$$
\begin{aligned}
\frac{\mu_{1}}{k_{t 1}^{\mathrm{TE}}} \tan \left(k_{t 1}^{\mathrm{TE}} d_{1}\right) & =-\frac{\mu_{2}}{k_{t 2}^{\mathrm{TE}}} \tan \left(k_{t 2}^{\mathrm{TE}} d_{2}\right) \\
\frac{\varepsilon_{1}}{k_{t 1}^{\mathrm{TM}}} \cot \left(k_{t 1}^{\mathrm{TM}} d_{1}\right) & =-\frac{\varepsilon_{2}}{k_{t 2}^{\mathrm{TM}}} \cot \left(k_{t 2}^{\mathrm{TM}} d_{2}\right) .
\end{aligned}
$$

Depending on the choice of material parameters, the above dispersion relations reveal interesting characteristics for the guided modes present in this waveguide. In the following sections, we will discuss some of the features of propagating guided modes for various pairs of ENG, MNG, DPS, and/or DNG slabs filling this guiding structure.

\section{PAIRS OF SNG SLABS: ENG-MNG, ENG-ENG, AND MNG-MNG PAIRS}

For a pair of ENG and MNG slabs, $\varepsilon_{1}<0, \mu_{1}>0, \varepsilon_{2}>0$, and $\mu_{2}<0$ and, thus, $k_{1}^{2}=\omega^{2} \mu_{1} \varepsilon_{1}<0$ and $k_{2}^{2}=\omega^{2} \mu_{2} \varepsilon_{2}<0$. 
For propagating modes, $\beta_{\mathrm{TE}}$ and $\beta_{\mathrm{TM}}$ should be real-valued quantities and, thus, we have $k_{\mathrm{ti}}=j \sqrt{\left|k_{i}\right|^{2}+\beta^{2}}$ purely imaginary for $i=1,2$. The dispersion relations (3) and (4) for the TE and TM modes can then be rewritten, respectively, as

$$
\begin{aligned}
& \frac{\mu_{1}}{\sqrt{\left|k_{1}\right|^{2}+\beta_{\mathrm{TE}}^{2}}} \tanh \left(\sqrt{\left|k_{1}\right|^{2}+\beta_{\mathrm{TE}}^{2}} d_{1}\right) \\
& =-\frac{\mu_{2}}{\sqrt{\left|k_{2}\right|^{2}+\beta_{\mathrm{TE}}^{2}}} \tanh \left(\sqrt{\left|k_{2}\right|^{2}+\beta_{\mathrm{TE}}^{2}} d_{2}\right) \\
& \frac{\varepsilon_{1}}{\sqrt{\left|k_{1}\right|^{2}+\beta_{\mathrm{TM}}^{2}}} \operatorname{coth}\left(\sqrt{\left|k_{1}\right|^{2}+\beta_{\mathrm{TM}}^{2}} d_{1}\right) \\
& =-\frac{\varepsilon_{2}}{\sqrt{\left|k_{2}\right|^{2}+\beta_{\mathrm{TM}}^{2}}} \operatorname{coth}\left(\sqrt{\left|k_{2}\right|^{2}+\beta_{\mathrm{TM}}^{2}} d_{2}\right) .
\end{aligned}
$$

For the case at hand where one slab is a lossless ENG and the other is a lossless MNG, our goal is to find the conditions under which we may obtain real-valued solutions for the longitudinal wavenumber $\beta$. Due to the monotonic behavior and asymptotic limit of the hyperbolic tangent and cotangent functions with real argument in (5) and (6), we expect to observe interesting dispersion characteristics. First, because (5) and (6) are indeed valid for any pair of SNG materials, we notice that if $\mu_{1}$ and $\mu_{2}$ have the same sign, (5) may not have any realvalued solution for $\beta_{\mathrm{TE}}$. Likewise, if $\varepsilon_{1}$ and $\varepsilon_{2}$ have the same sign, we may not have any TM mode with real $\beta_{\mathrm{TM}}$ from (6). This is not surprising since, for the case of the ENG-ENG or MNG-MNG pair, where permittivities and permeabilities have the same signs, i.e., $\varepsilon_{1} \varepsilon_{2}>0$ and $\mu_{1} \mu_{2}>0$, the wavenumber in the bulk paired materials is always imaginary. However, when we have a so-called "conjugate" pair, i.e., a pair of ENG and MNG slabs in this waveguide, both sides of (5) and (6) have the same sign and, thus, it is possible to have real-valued solutions for $\beta_{\mathrm{TE}}$ and $\beta_{\mathrm{TM}}$. This unusual feature is consistent with our previous findings on free-space plane-wave interaction with a pair of ENG-MNG slabs [44], in which we have shown the interesting "interface resonance" behavior between such two slabs. In order to gain some physical insights into the possible real-valued solutions of (5) and (6), let us assume that, for a given set of material parameters for the two slabs, the thickness of the ENG slab $d_{1}$ is known and fixed, and we then wish to find the MNG slab thickness $d_{2}$ such that $\beta$ attains a specific real value. This can be found by rearranging (5) and (6) as

$$
d_{2}^{\mathrm{TE}}=\frac{\tanh ^{-1}\left[\frac{\left|\mu_{1}\right| \sqrt{\left|k_{2}\right|^{2}+\beta_{\mathrm{TE}}^{2}}}{\left|\mu_{2}\right| \sqrt{\left|k_{1}\right|^{2}+\beta_{\mathrm{TE}}^{2}}} \tanh \left(\sqrt{\left|k_{1}\right|^{2}+\beta_{\mathrm{TE}}^{2}} d_{1}\right)\right]}{\sqrt{\left|k_{2}\right|^{2}+\beta_{\mathrm{TE}}^{2}}}
$$$$
d_{2}^{\mathrm{TM}}=\frac{\tanh ^{-1}\left[\frac{\left|\varepsilon_{2}\right| \sqrt{\left|k_{1}\right|^{2}+\beta_{\mathrm{TM}}^{2}}}{|| \varepsilon_{1} \mid \sqrt{\left|k_{2}\right|^{2}+\beta_{\mathrm{TM}}^{2}}} \tanh \left(\sqrt{\left|k_{1}\right|^{2}+\beta_{\mathrm{TM}}^{2}} d_{1}\right)\right]}{\sqrt{\left|k_{2}\right|^{2}+\beta_{\mathrm{TM}}^{2}}} .
$$

Obviously, a physical solution for $d_{2}$ exists only if the argument of the inverse hyperbolic tangent function in the above equations is between zero and unity, which suggests that not for every arbitrary pair of $d_{1}$ and $\beta$ we will be able to find a solution for $d_{2}$. However, when this condition is fulfilled, the solution for $d_{2}$ is unique due to the monotonic (nonperiodic) behavior of the hyperbolic tangent function. Specifically, for a given set of parameters for the pair of ENG and MNG slabs and a fixed $d_{1}$, there may only be one value for $d_{2}^{\mathrm{TE}}$ when a given real value for $\beta_{\mathrm{TE}}$ is desired and, similarly, there may only be one value for $d_{2}^{\mathrm{TM}}$ when $\beta_{\mathrm{TM}}$ is given. These features are in contrast with those of propagating modes in a conventional waveguide filled with a pair of DPS-DPS slabs, where one has multiple solutions for $d_{2}$ due to the periodic behavior of the fields in the transverse section. Furthermore, in the ENG-MNG waveguide, the field components vary as hyperbolic sinusoidal functions in the transverse plane, and they are mostly concentrated around the ENG-MNG interface. As will be shown in Section IV, similar field distributions and concentration near the interface may be observed in the DPS-DNG waveguides when $\beta>\max \left(\left|k_{1}\right|,\left|k_{2}\right|\right)$, as also observed by Nefedov and Tretyakov in [49]. There are other interesting properties in the DPS-DNG waveguides that may resemble those of the ENG-MNG waveguides discussed here. More will be mentioned on this similarity in Sections IV and V.

The conditions for having the arguments of the inverse hyperbolic tangent functions in (7) and (8) less than unity may be explicitly given as

$$
\begin{aligned}
\tanh \left(\sqrt{\beta_{\mathrm{TE}}^{2}+\left|k_{1}\right|^{2}} d_{1}^{\mathrm{TE}}\right) & <\frac{\left|\mu_{2}\right| \sqrt{\beta_{\mathrm{TE}}^{2}+\left|k_{1}\right|^{2}}}{\left|\mu_{1}\right| \sqrt{\beta_{\mathrm{TE}}^{2}+\left|k_{2}\right|^{2}}} \\
\tanh \left(\sqrt{\beta_{\mathrm{TM}}^{2}+\left|k_{1}\right|^{2}} d_{1}^{\mathrm{TM}}\right) & <\frac{\left|\varepsilon_{1}\right| \sqrt{\beta_{\mathrm{TM}}^{2}+\left|k_{2}\right|^{2}}}{\left|\varepsilon_{2}\right| \sqrt{\beta_{\mathrm{TM}}^{2}+\left|k_{1}\right|^{2}}} .
\end{aligned}
$$

If the terms on the right-hand side of (9) and (10) are greater than unity, then any values of $d_{1}^{T E}$ and $d_{1}^{T M}$, no matter how large or small, may satisfy these inequalities. However, if the right-hand terms are below the unity, then only certain limited ranges of $d_{1}^{\mathrm{TE}}$ and $d_{1}^{\mathrm{TM}}$ may fulfill (9) and (10). It is interesting to note that, due to the symmetric nature of the dispersion relations, when $d_{1}$ is limited to a finite range, $d_{2}$ will have a unique solution between zero and infinity and, vice versa, if $d_{2}$ is limited, then $d_{1}$ may find a unique solution in that infinite range. In other words, for any given $\beta$ (for TE or TM), only one of the two corresponding thicknesses $d_{1}$ and $d_{2}$ may be confined to a finite range of variation.

One special case, namely, when the right-hand side of (9) or (10) becomes unity, deserves a particular attention since, in such a case, neither $d_{1}$, nor $d_{2}$ is limited to a finite range of variation. The values of $\beta$ that may provide this special condition is explicitly given by

$$
\begin{aligned}
& \beta_{\mathrm{sw}}^{\mathrm{TE}}= \pm \omega \sqrt{\frac{\varepsilon_{1} / \mu_{1}-\varepsilon_{2} / \mu_{2}}{\mu_{1}^{-2}-\mu_{2}^{-2}}} \\
& \beta_{\mathrm{sw}}^{\mathrm{TM}}= \pm \omega \sqrt{\frac{\mu_{1} / \varepsilon_{1}-\mu_{2} / \varepsilon_{2}}{\varepsilon_{1}^{-2}-\varepsilon_{2}^{-2}}}
\end{aligned}
$$

where we recall that $\varepsilon_{1} \mu_{1}<0$ and $\varepsilon_{2} \mu_{2}<0$ for the ENG and MNG slabs. When, with proper choices of material parameters, $\beta_{\mathrm{sw}}^{\mathrm{TE}}$ or $\beta_{\mathrm{sw}}^{\mathrm{TM}}$ are real-valued quantities, they represent the wavenumbers for the TE or TM surface wave that may 
exist along the interface of the two semi-infinite lossless ENG and MNG media. These relations are formally similar to the wavenumber expressions for the surface waves supported at the interface of the DPS and DNG half-spaces described in [19] and [41]. (Strictly speaking, in the latter case, (11) and (12) represent the wavenumbers of surface waves only if these values of $\beta_{\mathrm{sw}}$ are real and greater than both $k_{1}$ and $k_{2}$ of the DPS and DNG media. In the ENG-MNG case, however, since $k_{1}$ and $k_{2}$ are both imaginary, as long as either of these $\beta_{\mathrm{sw}}$ is real, a surface wave may propagate.) From (11) and (12), the conditions for a given interface between ENG and MNG media to support a TE or TM surface wave can be expressed as

$$
\begin{gathered}
\text { TE: }\left\{\begin{array} { l } 
{ | \mu _ { 1 } | < | \mu _ { 2 } | } \\
{ | \eta _ { 1 } | > | \eta _ { 2 } | }
\end{array} \text { or } \left\{\begin{array}{l}
\left|\mu_{1}\right|>\left|\mu_{2}\right| \\
\left|\eta_{1}\right|<\left|\eta_{2}\right|
\end{array}\right.\right. \\
\text { TM: }\left\{\begin{array} { l } 
{ | \varepsilon _ { 1 } | < | \varepsilon _ { 2 } | } \\
{ | \eta _ { 1 } | < | \eta _ { 2 } | }
\end{array} \text { or } \left\{\begin{array}{l}
\left|\varepsilon_{1}\right|>\left|\varepsilon_{2}\right| \\
\left|\eta_{1}\right|>\left|\eta_{2}\right|
\end{array}\right.\right.
\end{gathered}
$$

where $\eta_{i}(i=1,2)$ denotes the intrinsic impedance of the medium, which is an imaginary quantity for ENG and MNG materials. As an aside, we note that conditions (13) imply that no interface may support both TE and TM surface waves, i.e., either a TE or a TM surface wave may be supported, but not both.

Fig. 2 presents the TE dispersion diagram of such an ENG-MNG waveguide for two different sets of material parameters. One striking feature of these diagrams, as already mentioned, is the single valueness of $d_{2}^{\mathrm{TE}}$ for a given set of $d_{1}$ and $\beta_{\mathrm{TE}}$. In order to understand these figures better, we first discuss some special limits.

\section{A. Thick Waveguides}

At one extreme, let us assume $\left|k_{1}\right| d_{1}$ and $\left|k_{2}\right| d_{2}$ to be large. Equations (5) and (6) may then be simplified as

$$
\begin{aligned}
& \frac{\left|\mu_{1}\right|}{\sqrt{\left|k_{1}\right|^{2}+\beta_{\mathrm{TE}}^{2}}} \simeq \frac{\left|\mu_{2}\right|}{\sqrt{\left|k_{2}\right|^{2}+\beta_{\mathrm{TE}}^{2}}} \\
& \frac{\left|\varepsilon_{1}\right|}{\sqrt{\left|k_{1}\right|^{2}+\beta_{\mathrm{TM}}^{2}}} \simeq \frac{\left|\varepsilon_{2}\right|}{\sqrt{\left|k_{2}\right|^{2}+\beta_{\mathrm{TM}}^{2}}}
\end{aligned}
$$

which are independent of the slab thicknesses. Solving these equations for $\beta_{\mathrm{TE}}$ and $\beta_{\mathrm{TM}}$, we obtain $\beta_{\mathrm{sW}}^{\mathrm{TE}}$ and $\beta_{\mathrm{sW}}^{\mathrm{TE}}$, as given in (11) and (12), respectively. This is physically justified since, for $\left|k_{1}\right| d_{1} \gg 1$ and $\left|k_{2}\right| d_{2} \gg 1$, the waveguide walls are far apart from the interface between the two slabs, and because the fields are concentrated around this interface, the ENG-MNG pair effectively behaves as two semi-infinite regions. Therefore, provided that a mode is supported by such a thick structure (and this depends on whether the interface may support a surface wave), this mode should resemble such a surface wave. This may be seen in Fig. 2(b), where we note that, in the limit of very large $\left|k_{1}\right| d_{1}$ and $\left|k_{2}\right| d_{2}$, the wavenumber $\beta_{\mathrm{TE}}$ approaches the value given in (11). It is worth noting that the curved line, beyond which the dispersion diagram in Fig. 2(b) "stops" and along which it diverges, is defined by the boundary of the region satisfying (9). Beyond this region, for a given pair of $d_{1}$ and $\beta$,

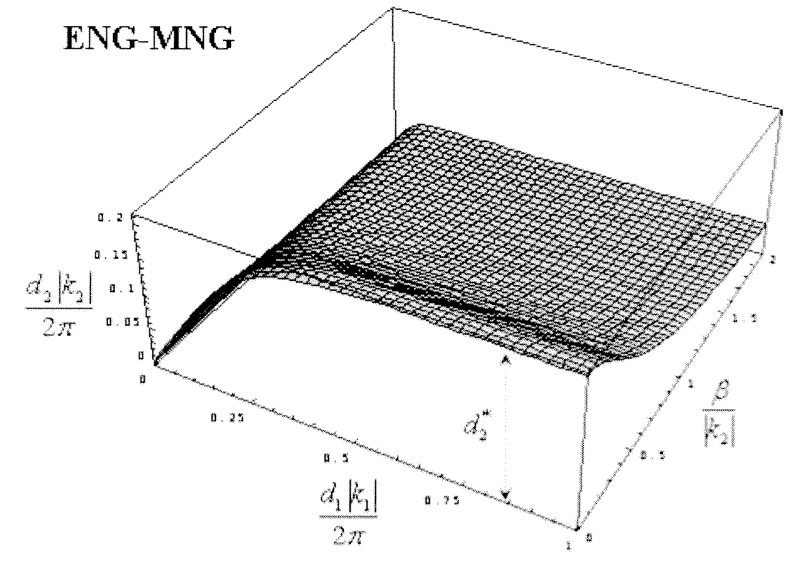

(a)

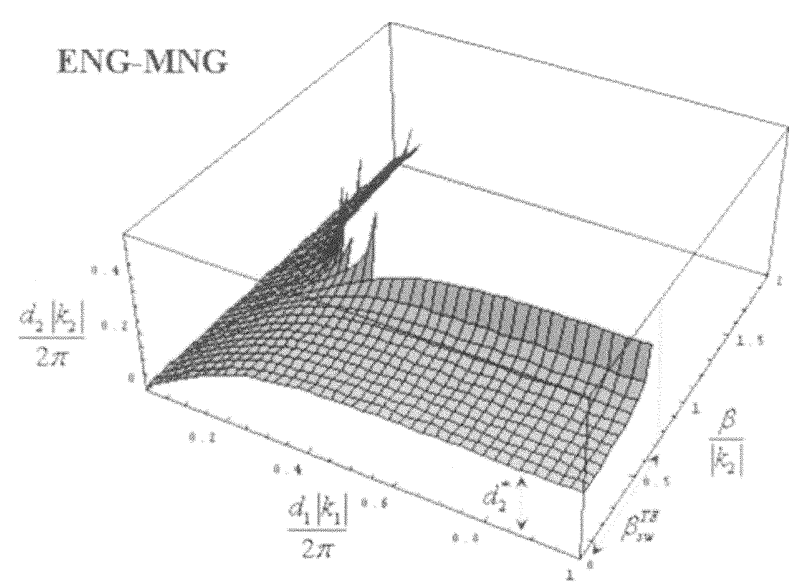

(b)

Fig. 2. Dispersion diagram for the $\mathrm{TE}$ mode in an ENG-MNG waveguide, illustrating the relationship among normalized $d_{1}, d_{2}$, and normalized real-valued $\beta_{\mathrm{TE}}$, as described in (7) for two sets of material parameters for a pair of ENG-MNG slabs at a given frequency: (a) when $\varepsilon_{1}=-2 \varepsilon_{0}, \mu_{1}=\mu_{0}, \varepsilon_{2}=3 \varepsilon_{0}, \mu_{2}=-2 \mu_{0}$ and (b) when $\varepsilon_{1}=-5 \varepsilon_{0}, \mu_{1}=2 \mu_{0}, \varepsilon_{2}=2 \varepsilon_{0}, \mu_{2}=-\mu_{0}$. The set of material parameters chosen in (a) does not allow a TE surface wave at the ENG-MNG interface, while the set chosen in (b) does. The value of $d_{2}^{*}$ is given by (18).

no real solution for $d_{2}$ may be obtained from (7). Analogous features may be observed for the TM case.

\section{B. Thin Waveguides}

If the thicknesses $\left|k_{1}\right| d_{1}$ and $\left|k_{2}\right| d_{2}$ are assumed to be very small, (3) and (4) may be approximated by

$$
\begin{aligned}
\gamma & \simeq-\frac{\mu_{2}}{\mu_{1}} \\
\beta_{\mathrm{TM}} & \simeq \pm \omega \sqrt{\frac{\mu_{1} \gamma+\mu_{2}}{\gamma / \varepsilon_{1}+1 / \varepsilon_{2}}}
\end{aligned}
$$

where $\gamma$ is a shorthand for $d_{1} / d_{2}$ and, obviously, should always be a positive quantity. We note the fact that these approximate expressions are valid for thin waveguides loaded with any pair of slabs since they have been obtained directly from (3) and (4). This point is physically justified considering the fact that, in thin waveguides, the transverse behavior of the field, which determines the possibility of a mode to propagate, is similar for DPS, DNG, and SNG materials since the hyperbolic and 
trigonometric sinusoidal functions have somewhat similar behavior in the limit of small arguments.

For a thin waveguide filled with a pair of DPS-DPS layers (and similarly with a pair of ENG-ENG, DPS-ENG, MNG-MNG, DNG-MNG, or DNG-DNG layers), (16) may never be satisfied because, for these pairs, $\mu_{2} / \mu_{1}>0$ and, thus, no TE mode may propagate in such a thin waveguide, as expected. On the other hand, (17) will provide the approximate value for $\beta_{\mathrm{TM}}$ of the dominant TM mode if $\beta_{\mathrm{TM}}$ turns out to be a real quantity for a given set of $\gamma$ and material parameters. We note that $\beta_{\mathrm{TM}}$ depends on the ratio of layer thicknesses, not on the total thickness. Therefore, this TM mode has no-cutoff thickness, i.e., there is not a thickness below which the TM mode may not propagate. For a DPS-DPS or DNG-DNG thin waveguide, this TM mode exists for any ratio $\gamma$, and its $\beta_{\mathrm{TM}}$ is sandwiched between $k_{1}$ and $k_{2}$, which are effectively the two limits of (17) for $\gamma \rightarrow \infty$ and $\gamma \rightarrow 0$, respectively. This implies that the TM field distribution in the transverse section of a DPS-DPS or DNG-DNG thin waveguide has to be expressed using the exponential functions in one of the two slabs (in the one with smaller wavenumber) and the sinusoidal functions in the other slab. The allowable ranges of variation of $\beta_{\mathrm{TM}}$ in (17) in terms of $\gamma$ are shown in Fig. 3 for various pairs of slabs. As shown in this figure, the ENG-MNG pair behaves differently: the existence of a no-cutoff dominant TM mode is restricted to the waveguides with $\gamma$ in the range between $\left|\varepsilon_{1}\right| /\left|\varepsilon_{2}\right|$ and $\left|\mu_{2}\right| /\left|\mu_{1}\right|$. However, its wavenumber $\beta_{\mathrm{TM}}$ is not restricted to any interval, i.e., an ENG-MNG waveguide may have a dominant no-cutoff TM mode with $\beta_{\mathrm{TM}}$ ranging from zero to infinity (Fig. 3).

Considering the TE case, a thin waveguide with a pair of ENG-MNG slabs (or also a pair of DNG-ENG, DPS-MNG, or DPS-DNG slabs) has $\mu_{2} / \mu_{1}<0$ and, thus, (16) may be satisfied for a certain value of $\gamma$. Equation (16) seems to be effectively independent of $\beta_{\mathrm{TE}}$. However, we should note that, in such a limit, the wavenumber $\beta_{\mathrm{TE}}$ of the guided mode may essentially attain any real value, as can be seen in Fig. 2(a) and (b) around the region where $\left|k_{1}\right| d_{1} \rightarrow 0$ and $\left|k_{2}\right| d_{2} \rightarrow 0$. In such a limit, no matter how thin these layers are [as long as they satisfy (16)], one (and only one) propagating mode may exist. In other words, this waveguide does not have a cutoff thickness for the TE modes. This feature represents a generalization of the analysis for the DPS-DNG thin cavity shown in [36]-[38]. For thin layers of ENG and MNG slabs, when $d_{1}$ and $d_{2}$ are selected to satisfy (16), the particular solution for $\beta_{\mathrm{TE}}$, which is unique, may be obtained by solving (5).

Another interesting feature to note in Fig. 2 is the relationship between $d_{1}$ and $d_{2}$ at $\beta=0$, which is the case of a one-dimensional cavity filled with the ENG-MNG pair. When $d_{1}$ is chosen to be large, $d_{2}$ will approach the finite limit given by

$$
d_{2}=\frac{1}{\left|k_{2}\right|} \tanh ^{-1}\left(\sqrt{\frac{\mu_{1} \varepsilon_{2}}{\mu_{2} \varepsilon_{1}}}\right)
$$

provided that $\sqrt{\left(\mu_{1} \varepsilon_{2}\right) /\left(\mu_{2} \varepsilon_{1}\right)}<1$ (which is the case for the parameters used in Fig. 2). This relation is obviously the same for both polarizations since their behaviors coincide when $\beta \rightarrow 0$. Depending on the choice of the material parameters,

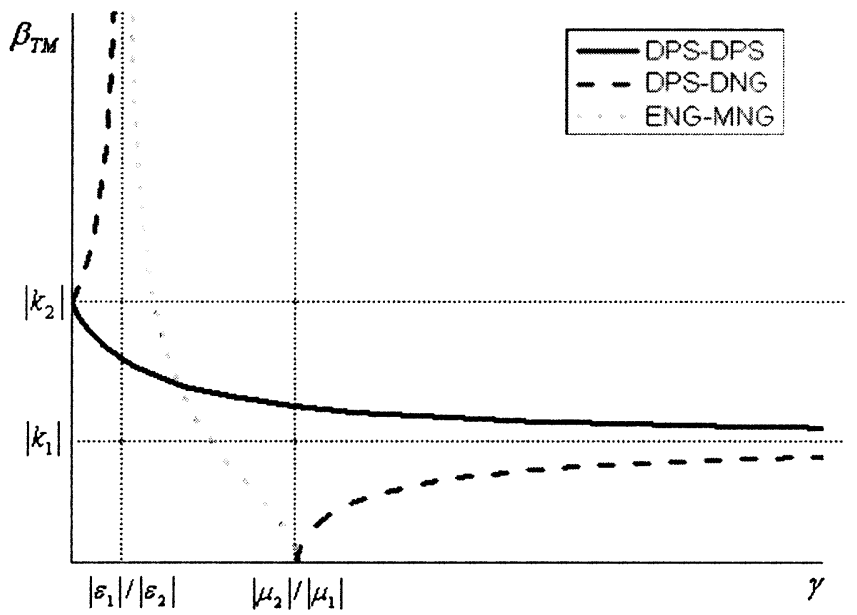

(a)

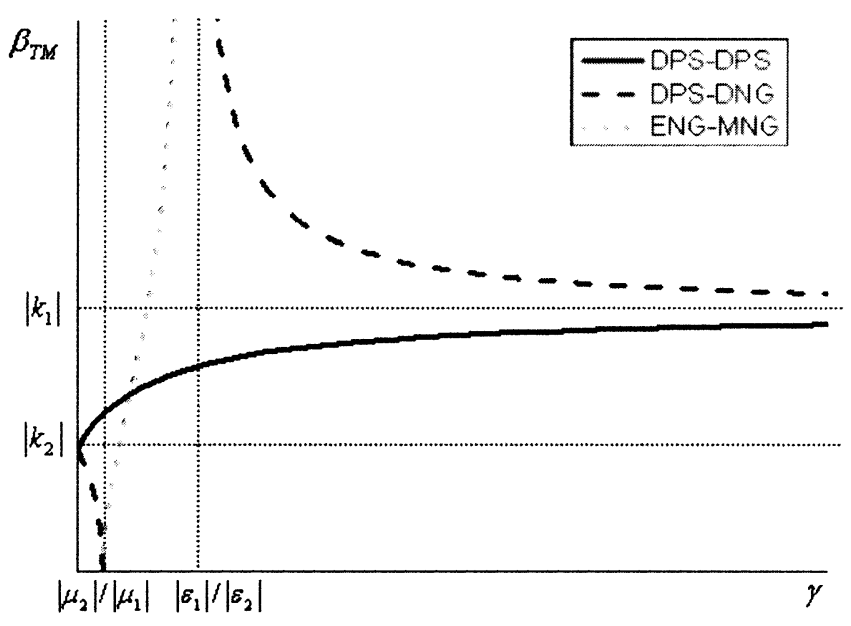

(b)

Fig. 3. $\beta_{\mathrm{TM}}$ of the dominant TM mode for thin waveguides filled with a pair of DPS-DPS, ENG-MNG, or DPS-DNG slabs versus $\gamma \equiv d_{1} / d_{2}$. In (a), the material parameters are chosen such that $\varepsilon_{1}= \pm 2 \varepsilon_{0}, \mu_{1}= \pm \mu_{0}, \varepsilon_{2}=$ $\pm 3 \varepsilon_{0}, \mu_{2}= \pm \mu_{0}$ for which $\left|k_{2}\right|>\left|k_{1}\right|$. In (b), the two slabs have been interchanged, i.e., slabs 1 and 2 in (a) are now slabs 2 and 1 in (b), respectively, thus, $\left|k_{2}\right|<\left|k_{1}\right|$. Here, we are concerned only with the positive real solutions for $\beta_{\mathrm{TM}}$, but its negative real solutions are simply obtained by flipping its sign.

for a fixed $d_{1}$, when we increase $\beta$ from zero the thickness $d_{2}$ usually does not show local minima or maxima, but it monotonically decreases or increases, as can be seen in Fig. 2(a) and (b), respectively. This feature implies the existence of a single mode in such an ENG-MNG waveguide for a given set of $d_{1}$ and $d_{2}$. It is important to point out that such a monomodal characteristic is effectively independent of the waveguide total thickness. For instance, from Fig. 2(a), we can see that, for a given $\beta_{\mathrm{TE}}$ and a specific allowable $d_{2}$, thickness $d_{1}$ may be chosen very large, resulting in a thick waveguide. However, still only one single mode is propagating in such a thick waveguide. This feature, not present in a conventional waveguide, may be potentially employed for possible applications in the design of monomodal waveguides with a large aperture. It can be shown that the monomodality property is present in any ENG-MNG waveguide, whose interface (between the ENG and MNG media) may support a surface wave [and, therefore, satisfies (13)] and in most (although not all) of the other ENG-MNG waveguides whose interface may not support a surface wave. 


\section{PAIRS OF DNG AND DPS SLABS: DPS-DNG, DPS-DPS, AND DNG-DNG PAIRS}

Here, we highlight some of the features of guided modes in the parallel-plate waveguides filled with various pairs of DPS and DNG layers, and then compare and contrast these features with those of the waveguides with SNG pairs mentioned in Section III. Here, for lossless DPS and DNG slabs, we have $\varepsilon \mu>0$ and, thus, $k_{i}^{2}=\omega^{2} \mu_{i} \varepsilon_{i}>0$ for $i=1,2$. First, we consider the special limit of "thin" waveguides.

\section{A. Thin Waveguides}

In many regards, as outlined above, various features of the thin DPS-DNG waveguide resemble those of the thin ENG-MNG waveguide. In fact, (16) and (17) again provide the approximate dispersion relations for the TE and TM modes in the DPS-DNG case. The TE polarization in this case is thoroughly equivalent with that of the thin ENG-MNG case. However, the TM mode here deserves further discussion. As may be seen from Fig. 3, the range of variation of $\beta_{\mathrm{TM}}$ in the thin DPS-DNG waveguide indeed differs from the ones in the thin ENG-MNG and standard DPS-DPS waveguides. Here, $\beta_{\mathrm{TM}}$ may attain values only outside the interval between $\left|k_{1}\right|$ and $\left|k_{2}\right|$ (effectively "complementary" to the standard DPS-DPS case, where $\beta_{\mathrm{TM}}$ is in this interval), and $\gamma$ should also be outside the range between $-\mu_{2} / \mu_{1}$ and $-\varepsilon_{1} / \varepsilon_{2}$. The fact that thin waveguides loaded with "conjugate" pairs of metamaterials (e.g., DPS-DNG or ENG-MNG) may support nonlimited $\beta_{\mathrm{TM}}$, may offer interesting possibilities in designing very thin resonant cavities, as already proposed in [36]-[38], for which $\beta=0$ when $\gamma=-\mu_{2} / \mu_{1}$, or for very thin waveguides having guided modes with high $\beta$. A similar observation regarding the possibility of $\beta_{\mathrm{TM}}$ to be very large has also been made by Nefedov and Tretyakov in [49].

\section{B. Waveguides With Arbitrary Thickness}

As mentioned earlier, the wavenumbers $k_{1}$ and $k_{2}$ assume real values in lossless DPS and DNG layers. Therefore, one may consider the following three distinct intervals for the longitudinal wavenumber $\beta$.

1) $\beta<\min \left(\left|k_{1}\right|,\left|k_{2}\right|\right)$ : In this interval, the transverse wavenumbers $k_{t 1}$ and $k_{t 2}$ are both real. Equations (3) and (4) may then be rearranged as follows to express the value of $d_{2}$ in terms of other parameters:

$$
\begin{aligned}
d_{2}^{\mathrm{TE}} & =\frac{\tan ^{-1}\left[-\frac{\mu_{1} \sqrt{k_{2}^{2}-\beta^{2}}}{\mu_{2} \sqrt{k_{1}^{2}-\beta^{2}}} \tan \left(\sqrt{k_{1}^{2}-\beta^{2}} d_{1}\right)\right]+m \pi}{\sqrt{k_{2}^{2}-\beta^{2}}} \\
d_{2}^{\mathrm{TM}} & =\frac{\tan ^{-1}\left[-\frac{\varepsilon_{2} \sqrt{k_{1}^{2}-\beta^{2}}}{\varepsilon_{1} \sqrt{k_{2}^{2}-\beta^{2}}} \tan \left(\sqrt{k_{1}^{2}-\beta^{2}} d_{1}\right)\right]+m \pi}{\sqrt{k_{2}^{2}-\beta^{2}}}
\end{aligned}
$$

where $m$ is an integer. From these equations, we note that, for a given set of material parameters, when $\beta$ and $d_{1}$ are fixed, the thickness $d_{2}$ for which a mode is supported has infinite solutions in DPS-DPS, DPS-DNG, and DNG-DNG waveguides.

2) $\min \left(\left|k_{1}\right|,\left|k_{2}\right|\right)<\beta<\max \left(\left|k_{1}\right|,\left|k_{2}\right|\right)$ : For $\left|k_{1}\right|<\beta<$ $\left|k_{2}\right|,(19)$ and (20) are modified as

$d_{2}^{\mathrm{TE}}=\frac{\tan ^{-1}\left[-\frac{\mu_{1} \sqrt{k_{2}^{2}-\beta^{2}}}{\mu_{2} \sqrt{\beta^{2}-k_{1}^{2}}} \tanh \left(\sqrt{\beta^{2}-k_{1}^{2}} d_{1}\right)\right]+m \pi}{\sqrt{k_{2}^{2}-\beta^{2}}}$

$d_{2}^{\mathrm{TM}}=\frac{\tan ^{-1}\left[\frac{\varepsilon_{2} \sqrt{\beta^{2}-k_{1}^{2}}}{\varepsilon_{1} \sqrt{k_{2}^{2}-\beta^{2}}} \tanh \left(\sqrt{\beta^{2}-k_{1}^{2}} d_{1}\right)\right]+m \pi}{\sqrt{k_{2}^{2}-\beta^{2}}}$.

(If $\left|k_{2}\right|<\beta<\left|k_{1}\right|$, similar expressions may be obtained, but in this case, $d_{2}^{\mathrm{TM}}$ and $d_{2}^{\mathrm{TE}}$ will be expressed in terms of inverse hyperbolic tangent functions and, thus, will be single-valued.) It should be mentioned that the minus sign in the argument of the inverse tangent functions has disappeared in (22). This is related to the fact that, in the thin waveguide approximation discussed earlier, a DPS-DPS waveguide may support a dominant no-cutoff TM mode in this range $\left(k_{1}<\beta_{\mathrm{TM}}<k_{2}\right)$, while a thin DPS-DNG waveguide may not (see Fig. 3). In fact, if we take the limit of (22) for $d_{1}$ to be very small, we will obtain $d_{2}^{\mathrm{TM}} \cong\left(\varepsilon_{2}\left(\beta^{2}-k_{1}^{2}\right)\right) /\left(\varepsilon_{1}\left(k_{2}^{2}-\beta^{2}\right)\right) d_{1}+(m \pi) /\left(\sqrt{k_{2}^{2}-\beta^{2}}\right)$ and, when $\varepsilon_{2} / \varepsilon_{1}<0$ (DPS-DNG case), the first admissible value for $m$ is unity, which implies that $d_{2}^{\mathrm{TM}}$ cannot be arbitrarily small and, thus, not allowing a dominant TM mode with $\beta_{\mathrm{TM}}$ in the range $\left(k_{1}<\beta_{\mathrm{TM}}<k_{2}\right)$ for a thin DPS-DNG waveguide.

Fig. 4(a) and (b) shows plots of the lowest admissible values of normalized $d_{2}$ from (19) and (21) for a given set of material parameters as a function of normalized $d_{1}$ and $\beta_{\mathrm{TE}}$ for the TE case. The parameters have been chosen so that $\left|k_{1}\right|<\left|k_{2}\right|$. (Analogous corresponding results may be obtained if $\left|k_{2}\right|<\left|k_{1}\right|$.) Panels (a) and (b) are for the DPS-DPS and DPS-DNG cases, respectively. It is important to reiterate that $d_{2}$ in (19)-(22) are multivalued for any given set of parameters and, thus, the plots of $d_{2}$ should be multibranched. (An example of such multibranched plots is shown in Fig. 4(c), which is for the DPS-DNG waveguide of Fig. 4(b).) However, Fig. 4(a) and (b) shows only the lowest positive values of $d_{2}$ for given parameters. The discontinuities observed are due to the jumps from one branch of $d_{2}$ to another in order to attain the lowest value for $d_{2}$; however, each branch of $d_{2}$ by itself is indeed continuous, as evident from Fig. 4(c). We can see from Fig. 4(a), where we have standard DPS-DPS waveguides, that as $d_{1} \rightarrow 0$, the lowest admissible value of $d_{2}$ to support a mode approaches a nonzero value of $\pi / k_{t 2}$, and this value expectedly becomes infinitely large as $\beta \rightarrow k_{2}$ since $k_{t 2} \rightarrow 0$. When $d_{2} \rightarrow 0$, on the other hand, the value of $d_{1}$ satisfies the relation $d_{1}=(p \pi) /\left(k_{t 1}\right)=(p \pi) /\left(\sqrt{k_{1}^{2}-\beta^{2}}\right)$, where $p$ is a positive integer. This locus can be clearly seen in Fig. 4. Thus, in a DPS-DPS waveguide, obviously no TE mode may exist if $d_{1}+d_{2}<\min \left((\pi) /\left(k_{t 1}\right),(\pi) /\left(k_{t 2}\right)\right)$, which implies that, as is well known, there is a constraint on the minimum 


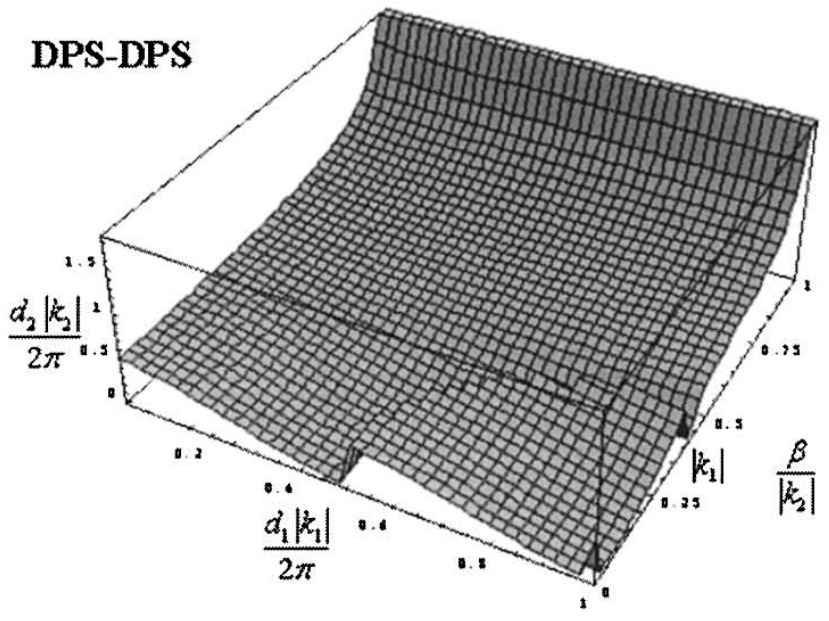

(a)

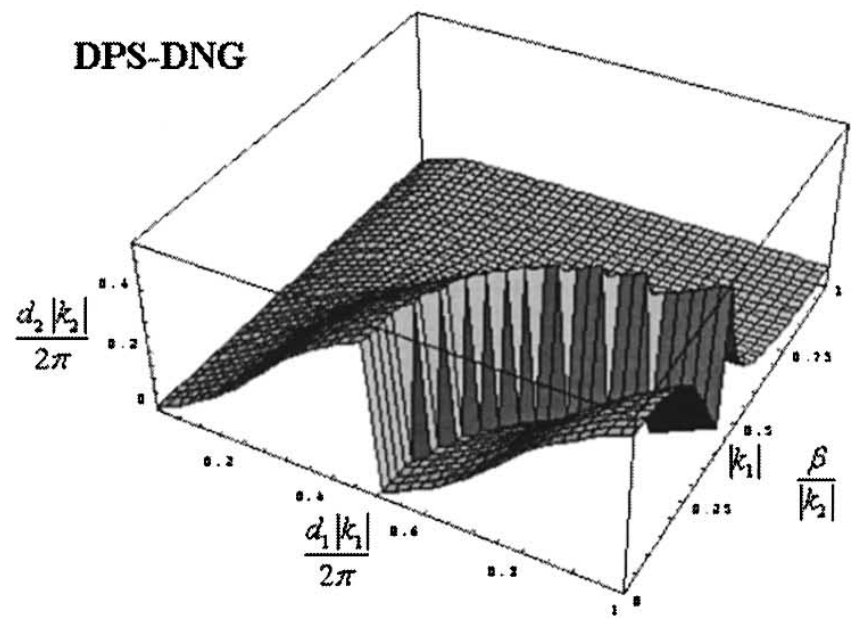

(b)

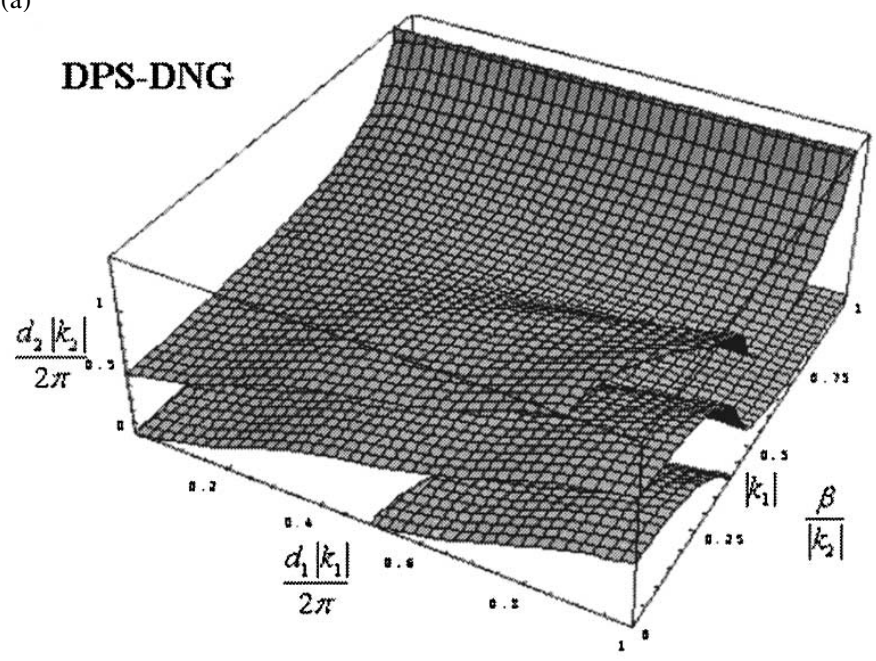

(c)

Fig. 4. Lowest admissible values of normalized $d_{2}$ as a function of normalized $d_{1}$ and $\beta$ for: (a) a DPS-DPS waveguide and (b) a DPS-DNG waveguide. $\varepsilon_{1}=2, \mu_{1}=1, \varepsilon_{2}= \pm 3, \mu_{2}= \pm 3$, TE case. In this case $k_{1}<k_{2}$. (c) The multibranched values of $d_{2}$ for the case in (b). (b) is essentially taken from (c), i.e., only the lowest values of $d_{2}$ are shown in (b).

total thickness of any DPS-DPS waveguide in order to have a TE mode. For DPS-DNG waveguides, however, the situation differs markedly: because a DNG slab may act as a phase compensator [36], effectively canceling the phase delay of a DPS slab, a DPS-DNG waveguide may have a TE mode as both $d_{1}$ and $d_{2}$ approach zero, as can be seen in Fig. 4(b) and (c). The constraint is on the ratio of the transverse phase delays in the two slabs, not on their sum. For the thin waveguide approximation, in a similar way, this constraint is manifested as the ratio $\gamma=d_{1} / d_{2}$, not the sum $d_{1}+d_{2}$, and it is described in (16) and (17). This is reflected in the slope of the curve in Fig. 4(b) in the vicinity of $d_{1} \rightarrow 0$ and $d_{2} \rightarrow 0$. The locus for $d_{2}=0$ has the same expression as in the DPS-DPS case, but here, $p$ in $d_{1}=p \pi / \sqrt{k_{1}^{2}-\beta^{2}}$ may also be zero. However, in Fig. 4(b), we note that as $\beta \rightarrow k_{2}-0$, the lowest positive value of $d_{2}$ does not become infinitely large. This can be understood by evaluating the limit of (21) for $\beta \rightarrow k_{2}-0$ as follows:

$$
\lim _{\beta \rightarrow k_{2}-0} d_{2}^{\mathrm{TE}}=-\frac{\mu_{1}}{\mu_{2}} \frac{\tanh \left(\sqrt{\beta^{2}-k_{1}^{2}} d_{1}\right)}{\sqrt{\beta^{2}-k_{1}^{2}}}+\frac{m \pi}{\sqrt{k_{2}^{2}-\beta^{2}}} .
$$

For a DPS-DNG waveguide, where $-\mu_{1} / \mu_{2}$ is a positive quantity, the smallest positive value for $d_{2}^{\mathrm{TE}}$ from (23) is obtained when $m=0$. This is, indeed, what we see in Fig. 4(b) in the neighborhood of $\beta \rightarrow k_{2}-0$. Upper branches of $d_{2}^{\mathrm{TE}}$ [not shown in Fig. 4(b), but shown in Fig. 4(c)], for which $m \geq 1$, approach $+\infty$ as $\beta \rightarrow k_{2}-0$ due to the term $(m \pi) /\left(\sqrt{k_{2}^{2}-\beta^{2}}\right)$. In the DPS-DPS case, the term $-\mu_{1} / \mu_{2}$ is a negative quantity and, therefore, the lowest value of $d_{2}^{\mathrm{TE}}$ in (23) is obtained when $m=1$, which causes $d_{2}^{\mathrm{TE}} \rightarrow \infty$ as $\beta \rightarrow k_{2}-0$ for every branch.

Another interesting information to obtain from Fig. 4 is the relationship between $d_{1}$ and $d_{2}$ when $\beta=0$. This " $\beta=0$ cut" in these figures represents the dispersion characteristics of a cavity resonator filled with a pair of DPS-DPS layers [see Fig. 4(a)] and DPS-DNG layers [see Fig. 4(b)]. The possibility of having a thin sub-wavelength cavity resonator with a pair of thin DPS and DNG slabs may be seen in Fig. 4(b) for $\beta=0$, as $d_{1} \rightarrow 0$ and $d_{2} \rightarrow 0$.

Fig. 5 presents the corresponding plots for the TM case. Similar to Fig. 4, in these plots, the lowest admissible values of normalized $d_{2}$ from (20) and (22) are shown as a function of nor- 


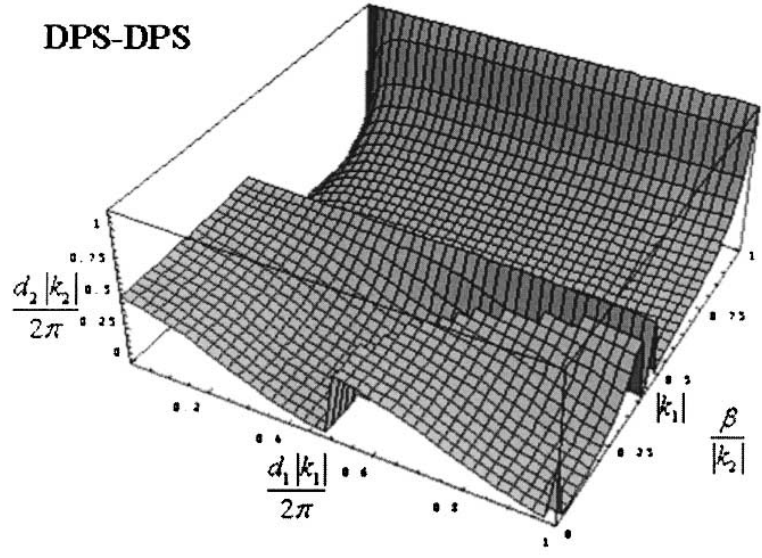

(a)

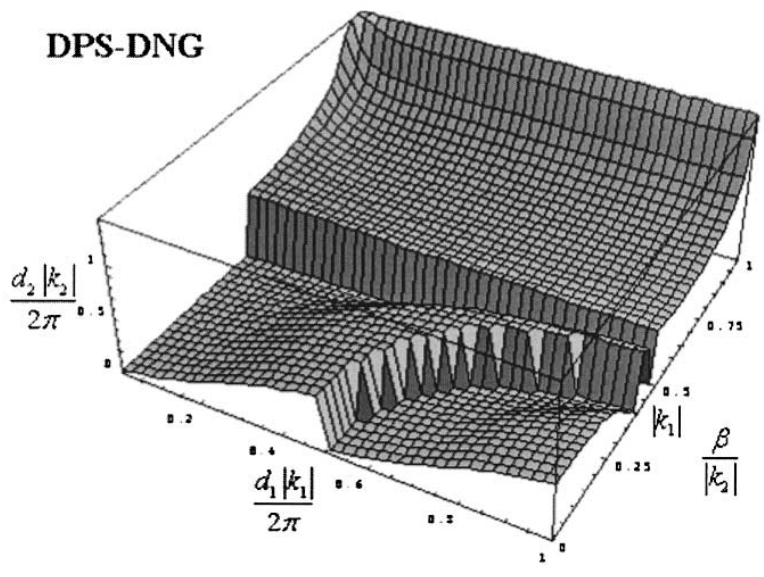

(b)

Fig. 5. Same as in Fig. 4, but TM polarization.

malized $d_{1}$ and $\beta_{\mathrm{TM}}$. The material parameters are the same as in Fig. 4, and are chosen such that $\left|k_{1}\right|<\left|k_{2}\right|$. Some of the main differences between the TE and TM modes can be observed by comparing these two figures. For instance, we note the expected presence of a no-cutoff dominant TM mode in the thin DPS-DPS waveguide in Fig. 5(a) for $d_{1} \rightarrow 0, d_{2} \rightarrow 0$, and $k_{1}<\beta_{\mathrm{TM}}<k_{2}$, and the absence of this mode in the thin DPS-DNG case in Fig. 5(b) in the range $k_{1}<\beta_{\mathrm{TM}}<k_{2}$. We also see the variation of $\gamma=d_{1} / d_{2}$ with $\beta_{\mathrm{TM}}$ in the limit of $d_{1} \rightarrow 0, d_{2} \rightarrow 0$ in the range $k_{1}<\beta_{\mathrm{TM}}<k_{2}$ for the DPS-DPS case in Fig. 5(a), and in the range $\beta_{\mathrm{TM}}<k_{1}$ for the DPS-DNG case in Fig. 5(b), all according to (17) and Fig. 3. In particular, we notice that, in thin DPS-DPS waveguides, the ratio $d_{1} / d_{2}$ attains all real values from zero to $+\infty$, as $\beta_{\mathrm{TM}}$ varies in the admissible interval between $k_{1}$ and $k_{2}$ [see Fig. 5(a)], whereas in the thin DPS-DNG case, when $k_{1}<k_{2}$ [see Fig. 5(b)], the ratio $d_{1} / d_{2}$ varies from $-\mu_{2} / \mu_{1}$ (when $\beta_{\mathrm{TM}}=0$ ) to $\infty$ (when $\beta_{\mathrm{TM}}=k_{1}$ ), and when $k_{1}>k_{2}$ (not shown here) the ratio $d_{1} / d_{2}$ goes from zero (when $\beta \rightarrow k_{2}$ ) to $-\mu_{2} / \mu_{1}$ (when $\beta_{\mathrm{TM}}=0$ ), all consistent with (17) and Fig. 3.

3) $\max \left(\left|k_{1}\right|,\left|k_{2}\right|\right)<\beta$ : In a conventional DPS-DPS waveguide, one may not have a guided mode with real-valued $\beta$ in this range. The same is true for a DNG-DNG waveguide. However, if one of the slabs is made of a lossless material with one and/or both of its permittivity and permeability negative, it will then be possible to have a TE and/or a TM guided mode, as shown below. For this range of $\beta$, the dispersion relations in (3) and (4) may be rewritten as follows:

$$
\begin{aligned}
d_{2}^{\mathrm{TE}} & =\frac{\tanh ^{-1}\left[-\frac{\mu_{1} \sqrt{\beta^{2}-k_{2}^{2}}}{\mu_{2} \sqrt{\beta^{2}-k_{1}^{2}}} \tanh \left(\sqrt{\beta^{2}-k_{1}^{2}} d_{1}\right)\right]}{\sqrt{\beta^{2}-k_{2}^{2}}} \\
d_{2}^{\mathrm{TM}} & =\frac{\tanh ^{-1}\left[-\frac{\varepsilon_{2} \sqrt{\beta^{2}-k_{1}^{2}}}{\varepsilon_{1} \sqrt{\beta^{2}-k_{2}^{2}}} \tanh \left(\sqrt{\beta^{2}-k_{1}^{2}} d_{1}\right)\right]}{\sqrt{\beta^{2}-k_{2}^{2}}} .
\end{aligned}
$$

These equations are analogous to (7) and (8) derived for the ENG-MNG waveguide, and they exhibit similar features. (The following section will give further insights into this analogy.) In both cases, $\beta^{2}>k_{i}^{2}$ with $i=1,2$ and the field distributions in the transverse plane are in terms of hyperbolic sinusoidal functions and, in both cases, $d_{2}$ is single-valued. The characteristics of the surface waves are also similar in both waveguides, and the formal expressions for $\beta_{\mathrm{SW}}^{\mathrm{TE}}$ and $\beta_{\mathrm{SW}}^{\mathrm{TM}}$ in (11) and (12) are still valid in the DPS-DNG case with appropriate values for the material parameters.

It can be shown that, at the interface of any given pair of semi-infinite DPS-DNG media, either a TE or a TM surface wave, but not both, may exist under the conditions

$$
\begin{gathered}
\text { TE: }\left\{\begin{array} { l } 
{ | \mu _ { 1 } | < | \mu _ { 2 } | } \\
{ | k _ { 1 } | > | k _ { 2 } | }
\end{array} \text { or } \left\{\begin{array}{l}
\left|\mu_{1}\right|>\left|\mu_{2}\right| \\
\left|k_{1}\right|<\left|k_{2}\right|
\end{array}\right.\right. \\
\text { TM: }\left\{\begin{array} { l } 
{ | \varepsilon _ { 1 } | < | \varepsilon _ { 2 } | } \\
{ | k _ { 1 } | > | k _ { 2 } | }
\end{array} \text { or } \left\{\begin{array}{l}
\left|\varepsilon_{1}\right|>\left|\varepsilon_{2}\right| \\
\left|k_{1}\right|<\left|k_{2}\right|
\end{array}\right.\right.
\end{gathered}
$$

which are more stringent than the corresponding conditions (13) obtained for SNG media since, in this case, we need to impose the additional constraint $\beta_{\mathrm{sw}}>\max \left(\left|k_{1}\right|,\left|k_{2}\right|\right)$. Fig. 6 presents the TE and TM cases for $\beta>k_{2}$ in the same waveguides of Figs. 4(b) and 5(b) filled with a pair of DPS and DNG slabs. Here again, the plots are single-branched since $d_{2}$ is singlevalued, similar to the ENG-MNG waveguide. For this choice of material parameters, no surface wave is supported. In Fig. 6(a), $\left(\left|\mu_{2}\right| \sqrt{\beta^{2}-k_{1}^{2}}\right) /\left(\left|\mu_{1}\right| \sqrt{\beta^{2}-k_{2}^{2}}\right)>1$ for any value of $\beta$ satisfying $\beta>k_{2}>k_{1}$, implying that, for any given set of $d_{1}$ and $\beta$, we can find a positive real-valued solution for $d_{2}$. It can be observed that, in the thin waveguide approximation, a TE mode exists, and the ratio $d_{1} / d_{2}$ again follows (16). Moreover, for any given $d_{1}$, the solution for $d_{2}$ is monotonically decreasing with $\beta$ in this region, suggesting that this DPS-DNG waveguide is monomodal regardless of its total thickness in this range, similar to the case of ENG-MNG waveguides described in Section III. In this case, however, generally the DPS-DNG waveguide may also support a finite number of modes with $\beta<k_{2}$. We note that, in Fig. 6(a), $d_{1}$ may attain any large value, still maintaining the monomodal property in this range, whereas $d_{2}$ is limited to a finite range to support this single mode. 


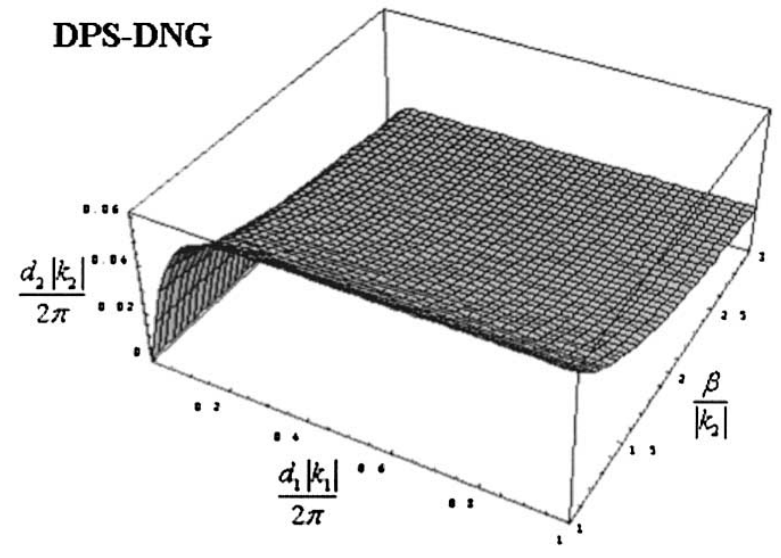

(a)

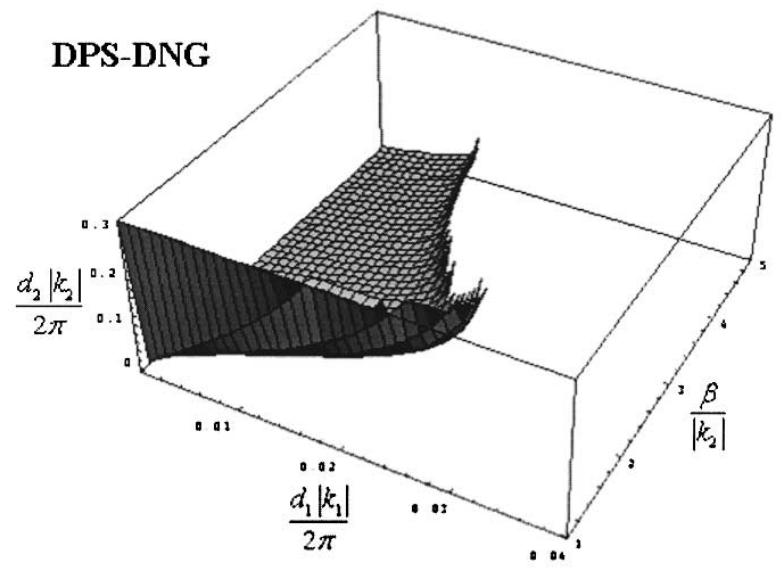

(b)

Fig. 6. (a) Normalized $d_{2}$ in terms of normalized $d_{1}$ and $\beta$, in the region $\beta>$ $k_{2}$, for TE modes in the waveguide filled with a pair of DPS and DNG slabs with material parameters given as shown in Fig. 4(b), where $k_{1}<k_{2}$. (b) The TM case. For this choice of material parameters, the DPS-DNG interface does not support a TE or TM surface wave.

Fig. 6(b) shows the plot for the TM polarization in the same DPS-DNG waveguide. In the thin waveguide limit, a no-cutoff TM mode is supported for all values of $\beta$ in the range $\beta>k_{2}$, following (17), and covering the other admissible values for $\gamma$ complementary to the range shown in Fig. 5(b) and in agreement with Fig. 3. The plot in Fig. 6(b) is only available in the region where the inequality (10) is fulfilled, beyond which $d_{2}$ does not have a physical solution. At the boundary, where

$$
\tanh \left(\sqrt{\beta^{2}-k_{1}^{2}} d_{1}^{\mathrm{TM}}\right)=\frac{\left|\varepsilon_{1}\right| \sqrt{\beta^{2}-k_{2}^{2}}}{\left|\varepsilon_{2}\right| \sqrt{\beta^{2}-k_{1}^{2}}}
$$

the value of $d_{2}$ approaches $+\infty$.

It is important to point out that, in Fig. 6(b), for a given set of $d_{1}$ and $d_{2}$, we may have two different modes supported in this range due to the nonmonotonic behavior of the boundary (27) in the $d_{1}-\beta$ plane. In other words, for a given set of material parameters and slab thicknesses, TM modes in this case may possess two distinct solutions for $\beta_{\mathrm{TM}}>\left|k_{2}\right|$. This anomalous behavior can be present only in waveguides filled with a pair of materials that do not support any surface wave at their interface, as is the case in Fig. 6(b). However, as can be seen from Fig. 6(a),

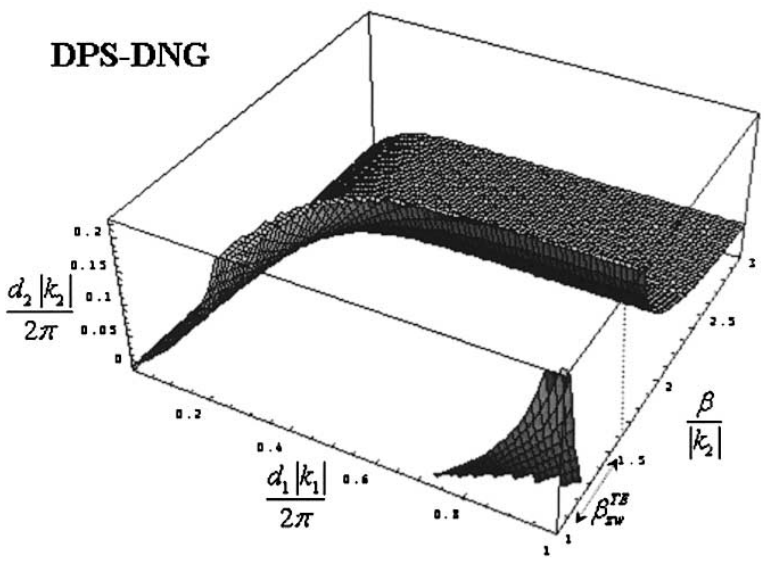

(a)

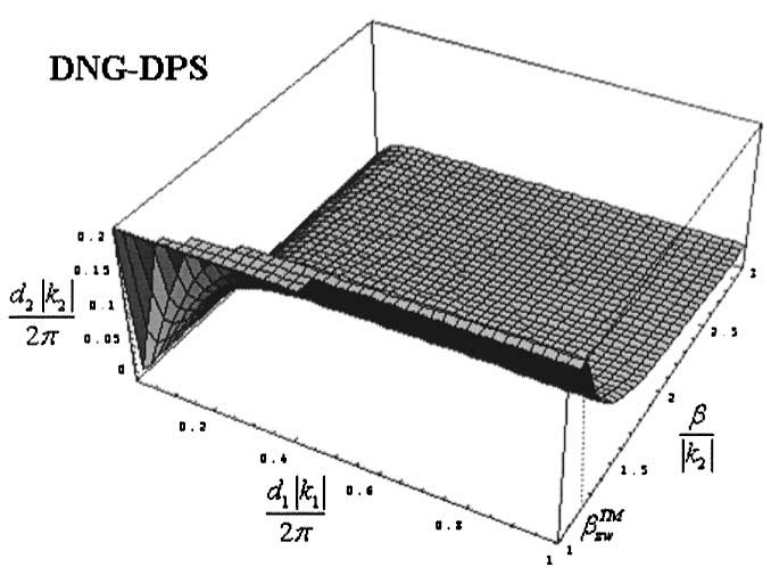

(b)

Fig. 7. (a) Normalized $d_{2}$ in terms of normalized $d_{1}$ and $\beta$, in the region $\beta>$ $k_{2}$, for TE modes in the waveguide filled with a pair of DPS and DNG slabs with material parameters $\varepsilon_{1}=3 \varepsilon_{0}, \mu_{1}=2 \mu_{0}, \varepsilon_{2}=-\varepsilon_{0}, \mu_{2}=-3 \mu_{0}$, where $k_{1}<k_{2}$. For this choice of material parameters, the DPS-DNG interface does support a TE surface wave. (b) Corresponding quantities for the TM modes in the waveguide filled with $\varepsilon_{1}=-2 \varepsilon_{0}, \mu_{1}=-\mu_{0}, \varepsilon_{2}=\varepsilon_{0}, \mu_{2}=3 \mu_{0}$, where $k_{1}<k_{2}$, for which the DPS-DNG interface does support a TM surface wave.

it is clear that the TE case is different: for any $d_{1}$, the behavior of $d_{2}$, in this case, is monotonic with $\beta$ since the solution for $d_{2}$ starts from a nonzero value and decreases monotonically to zero when $\beta \rightarrow \infty$.

When the interface can support a surface wave, the situation is different. Fig. 7(a) reveals corresponding information for the TE mode for $\beta>k_{2}$ in a DPS-DNG waveguide filled with materials that support a TE surface wave at their interface. (The region $\beta<k_{2}$ would be consistent with the results shown earlier.) In this case, however, the asymptote generated by the surface wave modifies the plots in the range $\beta>k_{2}$. The existence of a no-cutoff TE mode in the thin waveguide approximation is clearly shown in this figure near the region where $d_{1} \rightarrow 0$ and $d_{2} \rightarrow 0$. Condition (10) again provides the allowable region for given sets of $d_{1}$ and $\beta$. The monomodality in this range is also evident from this figure. The TM polarization for a DPS-DNG waveguide supporting a TM surface wave is shown in Fig. 7(b). Here also, the existence of the asymptotic behavior due to the surface wave provides a certain specific variation for $d_{2}^{\mathrm{TM}}$. In particular, we note that the monomodality in the range $\beta>\max \left(\left|k_{1}\right|,\left|k_{2}\right|\right)$ is present in this case. 


\section{More on the ANAlogy Between Modes IN THE ENG-MNG AND DPS-DNG WAVEGUIDES}

As seen in Sections III and IV, several properties of the modes with $\beta>\max \left(\left|k_{1}\right|,\left|k_{2}\right|\right)$ in DPS-DNG waveguides resemble the modal characteristics in ENG-MNG waveguides. The reason behind this similarity can be found in the transmission-line analogy for ENG, MNG, DNG, and DPS media discussed in [44] and [52]. In those papers, we have shown how, in many aspects, DPS and DNG media behave similarly to SNG materials when an evanescent wave is considered inside these media.

One may suggest the following heuristic transformations, which map a given SNG material [with parameters $(\varepsilon, \mu)$ ] with propagation wavenumber $\beta$ into an equivalent problem involving a DPS or DNG material with equivalent parameters $\left(\varepsilon^{\mathrm{eq}}, \mu^{\mathrm{eq}}\right)$ and $\beta_{\mathrm{eq}}$ :

$$
\begin{aligned}
& \left(\varepsilon_{\mathrm{ENG}}, \mu_{\mathrm{ENG}}, \beta^{2}\right) \rightarrow\left(\varepsilon_{\mathrm{DPS}}^{\mathrm{eq}}, \mu_{\mathrm{DPS}}^{\mathrm{eq}}, \beta_{\mathrm{eq}}^{2}\right) \\
& \equiv\left(-\varepsilon_{\mathrm{ENG}}, \mu_{\mathrm{ENG}}, \beta^{2}+2|k|^{2}\right)
\end{aligned}
$$

TE

$$
\begin{aligned}
& \left(\varepsilon_{\mathrm{MNG}}, \mu_{\mathrm{MNG}}, \beta^{2}\right) \rightarrow\left(\varepsilon_{\mathrm{DNG}}^{\mathrm{eq}}, \mu_{\mathrm{DNG}}^{\mathrm{eq}}, \beta_{\mathrm{eq}}^{2}\right) \\
& \quad \equiv\left(-\varepsilon_{\mathrm{MNG}}, \mu_{\mathrm{MNG}}, \beta^{2}+2|k|^{2}\right) \\
& \left(\varepsilon_{\mathrm{ENG}}, \mu_{\mathrm{ENG}}, \beta^{2}\right) \rightarrow\left(\varepsilon_{\mathrm{DNG}}^{\mathrm{eq}}, \mu_{\mathrm{DNG}}^{\mathrm{eq}}, \beta_{\mathrm{eq}}^{2}\right) \\
& \quad \equiv\left(\varepsilon_{\mathrm{ENG}},-\mu_{\mathrm{ENG}}, \beta^{2}+2|k|^{2}\right)
\end{aligned}
$$

TM :

$$
\begin{aligned}
& \left(\varepsilon_{\mathrm{MNG}}, \mu_{\mathrm{MNG}}, \beta^{2}\right) \rightarrow\left(\varepsilon_{\mathrm{DPS}}^{\mathrm{eq}}, \mu_{\mathrm{DPS}}^{\mathrm{eq}}, \beta_{\mathrm{eq}}^{2}\right) \\
& \quad \equiv\left(\varepsilon_{\mathrm{MNG}},-\mu_{\mathrm{MNG}}, \beta^{2}+2|k|^{2}\right) .
\end{aligned}
$$

In the above mapping, from SNG materials to DPS and DNG materials, $\beta^{2}$ always maps to values greater than $|k|^{2}$. These transformations suggest that for a given ENG-MNG waveguide with material parameters $\left(\varepsilon_{\mathrm{ENG}}, \mu_{\mathrm{ENG}}\right)$ and $\left(\varepsilon_{\mathrm{MNG}}, \mu_{\mathrm{MNG}}\right)$ and slab thicknesses $d_{1}$ and $d_{2}$, the behavior of the dispersion plot $d_{2}$ for any given set of $d_{1}$ and $\beta$ (for TE and TM polarizations) is equivalent to the one of a suitably designed DPS-DNG waveguided in the region $\beta>\max \left(\left|k_{1}\right|,\left|k_{2}\right|\right)$. It can be shown that, in this case, the mapping is given by the following conditions on the material parameters:

$$
\begin{aligned}
& \frac{\left|\mu_{\mathrm{DPS}}^{\mathrm{eq}}\right|}{\left|\mu_{\mathrm{DNG}}^{\mathrm{eq}}\right|}=\frac{\left|\mu_{\mathrm{ENG}}\right|}{\left|\mu_{\mathrm{MNG}}\right|} \\
& \begin{aligned}
& \frac{\left|\varepsilon_{\mathrm{DPS}}^{\mathrm{eq}}\right|}{\left|\varepsilon_{\mathrm{DNG}}^{\mathrm{eq}}\right|}=\frac{\left|\varepsilon_{\mathrm{ENG}}\right|}{\left|\varepsilon_{\mathrm{MNG}}\right|} \\
&\left|\varepsilon_{\mathrm{DNG}}^{\mathrm{eq}}\right|\left|\mu_{\mathrm{DNG}}^{\mathrm{eq}}\right|-\left|\varepsilon_{\mathrm{DPS}}^{\mathrm{eq}}\right|\left|\mu_{\mathrm{DPS}}^{\mathrm{eq}}\right| \\
&=\left|\varepsilon_{\mathrm{ENG}}\right|\left|\mu_{\mathrm{ENG}}\right|-\left|\varepsilon_{\mathrm{MNG}}\right|\left|\mu_{\mathrm{MNG}}\right|
\end{aligned}
\end{aligned}
$$

when the slab thicknesses remain the same as $d_{1}$ and $d_{2}$. The "equivalent" wavenumber for the guided mode in such a DPS-DNG waveguide may be expressed in terms of the parameters of the original ENG-MNG waveguide as

$$
\begin{aligned}
\beta_{\mathrm{eq}}^{2} & =\beta^{2}+\omega^{2}\left|\varepsilon_{\mathrm{ENG}}\right|\left|\mu_{\mathrm{ENG}}\right|+\omega^{2}\left|\varepsilon_{\mathrm{DPS}}^{\mathrm{eq}}\right|\left|\mu_{\mathrm{DPS}}^{\mathrm{eq}}\right| \\
& =\beta^{2}+\omega^{2}\left|\varepsilon_{\mathrm{MNG}}\right|\left|\mu_{\mathrm{MNG}}\right|+\omega^{2}\left|\varepsilon_{\mathrm{DNG}}^{\mathrm{eq}}\right|\left|\mu_{\mathrm{DNG}}^{\mathrm{eq}}\right|
\end{aligned}
$$

which is obviously greater than both $k_{1}^{\text {eq }}$ and $k_{2}^{\text {eq }}$ in the DPS and DNG slabs. An analogous "inverse transformation" may map any given DPS-DNG waveguide operating in the region $\beta>\max \left(\left|k_{1}\right|,\left|k_{2}\right|\right)$ to an equivalent ENG-MNG waveguide. From (29) and (30), some other analogies between the pairs of SNG layers with the pairs of DPS and DNG layers may be summarized as follows.

1) $E N G-E N G$ waveguides: for any $\beta$, their dispersion relations are equivalent to: a) (TE case), a DPS-DPS waveguide with $\beta^{2}>k_{i}^{2}(i=1,2)$ and, thus, no propagating mode is available and b) (TM case), a DNG-DNG waveguide with $\beta^{2}>k_{i}^{2}(i=1,2)$ and, again, no propagating mode may exist.

2) $M N G-M N G$ waveguides: for similar reasons, no propagating mode may exist in such waveguides.

3) $E N G-M N G$ waveguides: for any $\beta$, their dispersion relations are similar to the DPS-DNG waveguides with $\beta^{2}>k_{i}^{2}(i=1,2)$ for both polarizations. As shown in Sections III and IV, these waveguides may be designed to be monomodal, regardless of their total thickness.

4) DPS-ENG waveguides: when $\beta^{2}<k_{\mathrm{DPS}}^{2}$, their dispersion diagrams are equivalent to: a) (TE case), a DPS-DPS waveguide with $k_{1}^{2}<\beta^{2}<k_{2}^{2}$ and b) (TM case), a DPS-DNG waveguide with $k_{1}^{2}<\beta^{2}<k_{2}^{2}$. When $\beta^{2}>k_{\text {DPS }}^{2}$, they become equivalent to: a) (TE case), an ENG-MNG waveguide and b) (TM case), an MNG-MNG waveguide, both not supporting any propagating mode.

5) $D P S-M N G, D N G-E N G, D N G-M N G$ waveguides: corresponding results can be obtained by using duality. In particular, we note that the presence of a no-cutoff TM mode in a standard DPS-DPS waveguide implies $k_{1}^{2}<$ $\beta^{2}<k_{2}^{2}$, and this waveguide may then be regarded as a DPS-MNG waveguide, which allows no-cutoff solutions, following (17), for any $\beta<k_{\text {DPS }}$.

These and other analogies may be verified with the results of the previous sections. A set of analyses employing this transmission-line analogy as applied specifically to the waveguide geometries has been presented in [52].

\section{CONCLUSION}

In this paper, we have analyzed various properties of guided modes in parallel-plate waveguides filled with pairs of layers made of any two of the lossless ENG, MNG, DPS, and DNG materials. We have shown that, depending on the way of pairing these materials and the choice of the material parameters, one may obtain modal features that differ significantly from those of guided modes in conventional DPS-DPS waveguides. Among several important characteristics, we note 
the presence of TM and TE modes with no-cutoff thickness in the limit of thin DPS-DNG and ENG-MNG waveguides, the possibility of monomodality in thick ENG-MNG waveguides, and in DPS-DNG waveguides for slow modes with $\beta>\max \left(\left|k_{1}\right|,\left|k_{2}\right|\right)$, and the presence of modes with wavenumber $\beta$ greater than the wavenumbers of both layers. These features may provide potential applications in the design of novel devices and components, such as ultra-thin waveguides, thinner than diffraction-limited size, capable of supporting both TM and TE modes, single-mode thick fibers with less restriction and more flexibility on the fiber thickness, and very thin cavity resonators.

\section{REFERENCES}

[1] V. G. Veselago, "The electrodynamics of substances with simultaneously negative values of $\varepsilon$ and $\mu$," Sov. Phys.-Usp., vol. 10, no. 4, pp. 509-514, 1968. Russ. Usp. Fiz. Nauk, pp. 517-526, vol. 92, 1967.

[2] R. W. Ziolkowski and E. Heyman, "Wave propagation in media having negative permittivity and permeability," Phys. Rev. E, vol. 64, no. 5, 056625, 2001.

[3] R. A. Shelby, D. R. Smith, and S. Schultz, "Experimental verification of a negative index of refraction," Science, vol. 292, no. 5514, pp. 77-79, 2001.

[4] J. B. Pendry, A. J. Holden, D. J. Robbins, and W. J. Stewart, "Magnetism from conductors and enhanced nonlinear phenomena," IEEE Trans. Microwave Theory Tech., vol. 47, pp. 2075-2081, Nov. 1999.

[5] — - "Low-frequency plasmons in thin wire structures," J. Phys. Condensed Matter, vol. 10, pp. 4785-4809, 1998.

[6] D. R. Smith, W. J. Padilla, D. C. Vier, S. C. Nemat-Nasser, and S. Schultz, "Composite medium with simultaneously negative permeability and permittivity," Phys. Rev. Lett., vol. 84, no. 18, pp. 4184-4187, 2000.

[7] R. A. Shelby, D. R. Smith, S. C. Nemat-Nasser, and S. Schultz, "Microwave transmission through a two-dimensional, isotropic, left-handed metamaterial," Appl. Phys. Lett., vol. 78, no. 4, pp. 489-491, 2001.

[8] J. B. Pendry, "Negative refraction makes a perfect lens," Phys. Rev. Lett., vol. 85 , no. 18 , pp. 3966-3969, 2000.

[9] R. W. Ziolkowski, "Superluminal transmission of information through an electromagnetic metamaterial," Phys. Rev. E, vol. 63, no. 4, 046604, Apr. 2001.

[10] —, (2003, Apr.) Pulsed and CW Gaussian beam interactions with double negative metamaterial slabs. Opt. Express [Online], vol ((7)), pp. 662-681. Available: http://www.opticsexpress.org/abstract.cfm?URI=OPEX-11-7-662.

[11] — , "Tailoring the electromagnetic responses of metamaterials: Double negative medium designs and experiments," Proc. IEEE Nanotechnology, p. 223, Aug. 26-28, 2002.

[12] A. A. Oliner, "A periodic-structure negative-refractive-index medium without resonant elements," in IEEE AP-S Int. Symp./USNC/URSI Nat. Radio Science Meeting Dig., San Antonio, TX, June 16-21, 2002, p. 41 (of URSI Dig.).

[13] _ - "A planar negative-refractive-index medium without resonant elements," in IEEE MTT-S Int. Microwave Symp. Dig., Philadelphia, PA, June 8-13, 2003, pp. 191-194.

[14] A. Grbic and G. V. Eleftheriades, "A backward-wave antenna based on negative refractive index $L-C$ networks," Proc. IEEE AP-S Int Symp./USNC/URSI Nat. Radio Science Meeting, vol. 4, pp. 340-343, June 16-21, 2002.

[15] G. V. Eleftheriades, A. K. Iyer, and P. C. Kremer, "Planar negative refractive index media using periodically $L-C$ loaded transmission lines," IEEE Trans. Microwave Theory Tech., vol. 50, pp. 2702-2712, Dec. 2002.

[16] C. Caloz, H. Okabe, T. Iwai, and T. Itoh, "Transmission line approach of left-handed materials," in IEEE AP-S Int. Symp./USNC/URSI Nat. Radio Science Meeting Dig., San Antonio, TX, June 16-21, 2002, p. 39 (of URSI Dig.)

[17] L. Liu, C. Caloz, C.-C. Chang, and T. Itoh, "Forward coupling phenomena between artificial left-handed transmission lines," J. Appl. Phys., vol. 92, no. 9, pp. 5560-5565, Nov. 1, 2002.

[18] Z. M. Zhang and C. J. Fu, "Unusual photon tunneling in the presence of a layer with a negative refractive index," Appl. Phys. Lett., vol. 80, no. 6, pp. 1097-1099, Feb. 11, 2002.
[19] I. V. Lindell, S. A. Tretyakov, K. I. Nikoskinen, and S. Ilvonen, "BW media-Media with negative parameters, capable of supporting backward waves," Microwave Opt. Technol. Lett., vol. 31, no. 2, pp. 129-133, 2001.

[20] S. A. Tretyakov, "Metamaterials with wideband negative permittivity and permeability," Microwave Opt. Technol. Lett., vol. 31, no. 3, pp. 163-165, 2001.

[21] K. G. Balmain, A. A. E. Luttgen, and P. C. Kremer, "Resonance cone formation, reflection, refraction, and focusing in a planar anisotropic metamaterial," IEEE Antennas Wireless Propagat. Lett., vol. 1, pp. 146-149, July 2002.

[22] M. W. McCall, A. Lakhtakia, and W. S. Weiglhofer, "The negative index of refraction demystified," Eur. J. Phys., vol. 23, pp. 353-359, 2002.

[23] N. Garcia and M. Nieto-Vesperinas, "Left-handed materials do not make a perfect lens," Phys. Rev. Lett., vol. 88, no. 20, 207403, 2002.

[24] A. Lakhtakia, "Reversed circular dichroism of isotropic chiral mediums with negative permeability and permittivity," Microwave Opt. Technol. Lett., vol. 33, no. 2, pp. 96-97, Apr. 20, 2002.

[25] A. N. Lagarkov and V. N. Kisel, "Electrodynamics properties of simple bodies made of materials with negative permeability and negative permittivity," Dokl. Phys., vol. 46, no. 3, pp. 163-165, 2001. Russian Dokl. Akad. Nauk SSSR, pp. 40-43, vol. 377, no. 1, 2001.

[26] M. W. Feise, P. J. Bevelacqua, and J. B. Schneider, "Effects of surface waves on behavior of perfect lenses," Phys. Rev. B, Condens. Matter, vol. 66, 035113, 2002.

[27] R. Marques, F. Medina, and R. Rafii-El-Idrissi, "Role of bianisotropy in negative permeability and left-handed metamaterials," Phys. Rev. B, Condens. Matter, vol. 65, no. 14, 144440, 2002.

[28] R. Marques, J. Martel, F. Mesa, and F. Medina, "A new 2-D isotropic left-handed metamaterial design: Theory and experiment," Microwave Opt. Technol. Lett., vol. 36, pp. 405-408, Dec. 2002.

[29] S. Hrabar and J. Bartolic, "Simplified analysis of split ring resonator used in backward metamaterials," in Proc. Int. Mathematical Methods in Electromagnetic Theory Conf., Kiev, Ukraine, Sept. 10-13, 2002, pp. $560-562$.

[30] P. M. Valanju, R. M. Walser, and A. P. Valanju, "Wave refraction in negative-index media: Always positive and very inhomogeneous," Phys. Rev. Lett., vol. 88, no. 18, 012220, 2002.

[31] D. R. Smith, D. Schurig, and J. B. Pendry, "Negative refraction of modulated electromagnetic waves," Appl. Phys. Lett., vol. 81, no. 15, pp. 2713-2715, Oct. 7, 2002.

[32] P. Gay-Balmaz and O. J. F. Martin, "Efficient isotropic magnetic resonators," Appl. Phys. Lett., vol. 81, no. 5, pp. 939-941, July 29, 2002.

[33] J. A. Kong, B.-I. Wu, and Y. Zhang, "A unique lateral displacement of a Gaussian beam transmitted through a slab with negative permittivity and permeability," Microwave Opt. Technol. Lett., vol. 33, no. 2, pp. 136-139, 2002.

[34] R. A. Silin and I. P. Chepurnykh, "On media with negative dispersion," J. Commun. Technol. Electron., vol. 46, no. 10, pp. 1121-1125, 2001.

[35] — , "On media with negative dispersion" (in Russian), Radiotekh., vol. 46, no. 10, pp. 1212-1217, 2001.

[36] N. Engheta. (2002) An idea for thin subwavelength cavity resonators using metamaterials with negative permittivity and permeability. IEEE Antennas Wireless Propagat. Lett. [Online], vol (1), pp. 10-13. Available: http://ieeexplore.ieee.org/Xplore/RecentIssue.jsp?Punumber=7727.

[37] N. Engheta, "Guided waves in paired dielectric-metamaterial with negative permittivity and permeability layers," presented at the USNC-URSI Nat. Radio Science Meeting URSI Dig., Boulder, CO, Jan. 9-12, 2002, p. 66.

[38] — , "Ideas for potential applications of metamaterials with negative permittivity and permeability," in Advances in Electromagnetics of Complex Media and Metamaterials. ser. NATO Sci., S. Zouhdi, A. H. Sihvola, and M. Arsalane, Eds. Norwell, MA: Kluwer, 2002, pp. 19-37.

[39] A. Alù and N. Engheta, "Mode excitation by a line source in a parallelplate waveguide filled with a pair of parallel double-negative and doublepositive slabs," in Proc. IEEE AP-S Int. Symp., vol. 3, Columbus, OH, June 22-27, 2003, pp. 359-362.

[40] _ "Anomalous mode coupling in guided-wave structures containing metamaterials with negative permittivity and permeability," in Proc. IEEE Nanotechnology, Washington, DC, Aug. 26-28, 2002, pp. 233-234.

[41] $~$ "Radiation from a traveling-wave current sheet at the interface between a conventional material and a material with negative permittivity and permeability," Microwave Opt. Technol. Lett., vol. 35, no. 6, pp. 460-463, Dec. 20, 2002. 
[42] N. Engheta, "Is Foster's reactance theorem satisfied in double-negative and single-negative media?," Microwave Opt. Technol. Lett., vol. 39, no. 1 , pp. 11-14, Oct. 5, 2003.

[43] N. Engheta, S. Nelatury, and A. Hoorfar, "The role of geometry of inclusions in forming metamaterials with negative permittivity and permeability," in Proc. XXVII Gen. Assembly Int. Union of Radio Science, Maastricht, The Netherlands, Aug. 17-24, 2002, Paper 1935 (on the CD).

[44] A. Alù and N. Engheta, "Pairing an epsilon-negative slab with a mu-negative slab: Resonance, tunneling and transparency," IEEE Trans. Antennas and Propagation (Special Issue), vol. 51, pp. 2558-2571, Oct. 2003.

[45] _ - "Mono-modal waveguides filled with a pair of parallel epsilonnegative (ENG) and mu-negative (MNG) metamaterial layers," in IEEE MTT-S Int. Microwave Symp. Dig., Philadelphia, PA, June 8-13, 2003, pp. 313-316.

[46] D. R. Fredkin and A. Ron, "Effective left-handed (negative index) composite material," Appl. Phys. Lett., vol. 81, no. 10, pp. 1753-1755, Sept. 2,2002

[47] A. Topa, "Contradirectional interaction in a NRD waveguide coupler with a metamaterial slab," in XXVII International Union of Radio Science Gen. Assembly, Maastricht, The Netherlands, Aug. 17-24, 2002, Paper 1878 (on the CD).

[48] I. S. Nefedov and S. A. Tretyakov, "Theoretical study of waveguiding structures containing backward-wave materials," in XXVII International Union of Radio Science Gen. Assembly, Maastricht, The Netherlands, Aug. 17-24, 2002, Paper 1074 (on the CD).

[49] - (2002, Nov. 10) Waveguide containing a backward-wave slab [Online] e-print in arXiv:cond-mat/0 211185 v1, http://arxiv.org/pdf/condmat/0211 185 .

[50] B.-I. Wu, T. M. Grzegorczyk, Y. Zhang, and J. A. Kong, "Guided modes with imaginary transverse wave number in a slab waveguide with negative permittivity and permeability," J. Appl. Phys., vol. 93, no. 11, pp. 9386-9388, June 1, 2003.

[51] C. Caloz, C.-C. Chang, and T. Itoh, "Full-wave verification of the fundamental properties of left-handed materials in waveguide configurations," J. Appl. Phys., vol. 90, no. 11, pp. 5483-5486, Dec. 2001.

[52] A. Alù and N. Engheta, "Distributed-circuit-element description of guided-wave structures and cavities involving double-negative or single-negative media," in Proc. SPIE, vol. 5218, San Diego, CA, Aug. 4-5, 2003, pp. 145-155.

[53] C. H. Papas, Theory of Electromagnetic Wave Propagation. New York: Dover, 1988.

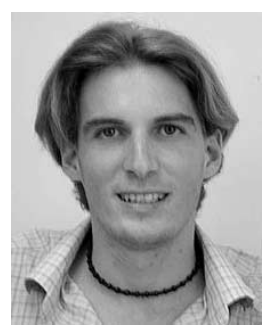

Andrea Alù was born in Rome, Italy, on September 27, 1978. He received the Electronic Engineering Laurea degree (summa cum laude) from the University of "Roma Tre," Rome, Italy in 2001, and is currently working toward the M.S. degree at the University of "Roma Tre."

In 2002, he was a visiting graduate student with the Moore School of Electrical Engineering, University of Pennsylvania, Philadelphia, where he was involved with metamaterial applications to waveguides and resonators at microwave and optical frequencies. His current research interests are in the areas of wave interaction with metamaterials and integrated planar and conformal circuits and antennas loaded by complex materials.

Mr. Alù was the recipient of the Isabella Sassi Bonadonna Scholarship presented by the Italian Electrical Association (AEI) in 2001. He was also the recipient of the Scipione Bobbio Award presented by the Fondazione IDIS - Città della Scienza in 2002, and of the Second Prize in the IEEE Antennas and Propagation Society (IEEE AP-S) Student Paper Contest in 2003.

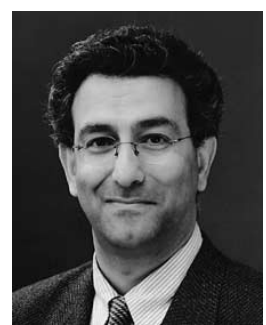

Nader Engheta (S'80-M'82-SM'89-F'96) received the B.S. degree in electrical engineering from the University of Tehran, Tehran, Iran, in 1978, and the M.S. and Ph.D. degrees from the California Institute of Technology (Caltech), Pasadena, in 1979 and 1982, respectively, both in electrical engineering.

From June 1982 to June 1983, he was a Post-Doctoral Research Fellow with Caltech. From June 1983 to June 1987, he was a Senior Research Scientist with the Dikewood Division, Kaman Sciences Corporation, Santa Monica, CA. In July 1987, he joined the faculty of the University of Pennsylvania, where he is currently a Professor of electrical and systems engineering. He is also a member of the David Mahoney Institute of Neurological Sciences, University of Pennsylvania, and a member of the Bioengineering Graduate Group, University of Pennsylvania. He was the Graduate Group Chair of electrical engineering from July 1993 to June 1997. His research interests and activities are in the areas of fields and waves phenomena, metamaterials and complex media, bio-inspired/biomimetic polarization imaging and polarization vision, plasmonic optics, nano-electromagnetics and optics, small antennas for wireless applications, bio-inspired hyperspectral sensing, biologically based visualization and physics of sensing and display of polarization information, through-wall microwave imaging, electromagnetics/electrophysics of brain cortical potentials [e.g., electroencephalography (EEG)], fractional operators, and fractional paradigm in electrodynamics. He was an Associate Editor for Radio Science (1991-1996) and was on the Editorial Board of the Journal of Electromagnetic Waves and Applications.

Dr. Engheta is a Guggenheim Fellow and a Fellow of the Optical Society of America. He is a member of the American Physical Society (APS), the American Association for the Advancement of Science (AAAS), Sigma Xi, Commissions B and D of the U.S. National Committee (USNC) of the International Union of Radio Science (URSI), and a member of the Electromagnetics Academy. $\mathrm{He}$ is an associate editor of the IEEE ANTENNAS AND WIRELESS PROPAGATION LETTERS (2002-present), and was an associate editor for the IEEE TRansactions on Antennas and Propagation (1996-2001). He served as an IEEE Antennas and Propagation Society Distinguished Lecturer (1997-1999). He was the chair (1989-91) and vice-chair (1988-89) of the joint chapter of the IEEE Antennas and Propagation Society (IEEE AP-S)/Microwave Theory and Techniques Society (IEEE MTT-S) in the Philadelphia Section. He is an elected member of the Administrative Committee (AdCom) of the IEEE AP-S since January 2003. He has guest edited/co-edited several special issues, namely, the special issue on "Wave Interaction with Chiral and Complex Media" of the Journal of Electromagnetic Waves and Applications (1992), the partial special issue on "Antennas and Microwaves of the Journal of the Franklin Institute (from the 13th Annual Benjamin Franklin Symposium) (1995), the special issue "Electrodynamics in Complex Environments" of the Wave Motion (with L. B. Felsen) (2001), and the special issue on "Metamaterials" of the IEEE TRANSACTIONS ON ANTENNAS AND PROPAGATION (with R. W. Ziolkowski) (2003). He was a recipient of the IEEE Third Millennium Medal. He was the recipient of numerous awards and distinctions for his scholarly research contributions and teaching activities including the UPS Foundation Distinguished Educator term Chair (July 1999-June 2000), the Fulbright Naples Chair Award for Naples, Italy (1998), a National Science Foundation (NSF) Presidential Young Investigator (PYI) Award (1989), the S. Reid Warren, Jr. Award for distinguished teaching from the School of Engineering and Applied Science, University of Pennsylvania (1993 and 2002), the Christian F. and Mary R. Lindback Foundation Award (1994), and the W. M. Keck Foundation's Engineering Teaching Excellence Award (1995). 